\title{
Sobolev, Besov and Paley-Wiener vectors in Banach and Hilbert spaces
}

\author{
Isaac Z. Pesenson \\ Dedicated to 100th Birthday of my teacher S.G. Krein.
}

\begin{abstract}
We consider Banach spaces equipped with a set of strongly continuous bounded semigroups satisfying certain conditions. Using these semigroups we introduce an analog of a modulus of continuity and define analogs of Besov norms. A generalization of a classical interpolation theorem is proven in which the role of Sobolev spaces is played by subspaces defined in terms of infinitesimal operators of these semigroups. We show that our assumptions about a given set of semigroups are satisfied in the case of a strongly continuous bounded representation of a Lie group. In the case of a unitary representation in a Hilbert space we consider an analog of the Laplace operator and use it to define Paley-Wiener vectors. It allows us to develop a generalization of the Shannon-type sampling in Paley-Wiener subspaces and to construct PaleyWiener nearly Parseval frames in the entire Hilbert space. It is shown that Besov spaces defined previously in terms of the modulus of continuity can be described in terms of approximation by Paley-Wiener vectors and also in terms of the frame coefficients. Throughout the paper we extensively use theory of interpolation and approximation spaces. The paper ends with applications of our results to function spaces on homogeneous manifolds.
\end{abstract}

\section{Introduction and Main Results}

I am honored to have had Selim Grigorievich Krein as my academic advisor and co-author. Without his steady, strong interest in my research and his support I would not have been able to have the privilege of becoming a mathematician. His inspiring encouragement shaped not only my professional trajectory but my life in general.

The first five sections of this paper are devoted to Sobolev and Besov subspaces and to relevant moduli of continuity in Banach spaces. This theory is rooted in my results obtained in 70s: [19-21, 23, 9]. It is a far going generalization of the one-dimensional theory by J. Lions 11 and J. Lions-J. Peetre 12 which I learned from the nice book by P. Butzer and H. Berens [3. The Paley-Wiener

2000 Mathematics Subject Classification. Primary 43A85, 41A17.

Key words and phrases. Groups and semigroups of operators, Sobolev, Besov, and PaleyWiener vectors, Interpolation and Approximation spaces.

I would like to thank Dr. Meyer Pesenson for a number of useful suggestions. 
vectors and corresponding approximation theory in abstract Hilbert spaces were introduced in 80s in my papers [22, [24, 25, 9] (see sections 6] and 7] below). In papers [26-34] I used the notion of Paley-Wiener vectors to prove Shannon-type sampling theorems on manifolds and in general Hilbert spaces. The construction of frames and description of Besov subspaces on manifolds and in Hilbert spaces in terms of frame coefficients is rather recent development and can be found in [7], 39. Some of these results were summarized in [6].

We consider a Banach space $\mathbf{E}$ and operators $D_{1}, D_{2}, \ldots, D_{d}$ which generate strongly continuous uniformly bounded semigroups $T_{1}(t), T_{2}(t), \ldots, T_{d}(t),\|T(t)\| \leq$ $1, t \geq 0$. An analog of a Sobolev space is introduced as the space $\mathbf{E}^{r}$ of vectors in $\mathbf{E}$ for which the following norm is finite

$$
\|f\|_{\mathbf{E}^{r}}=\|f\|_{\mathbf{E}}+\sum_{k=1}^{r} \sum_{1 \leq j_{1}, \ldots j_{k} \leq d}\left\|D_{j_{1}} \ldots D_{j_{k}} f\right\|_{\mathbf{E}},
$$

where $r \in \mathbb{N}, f \in \mathbf{E}$. By using the closed graph theorem and the fact that each $D_{i}$ is a closed operator in $\mathbf{E}$, one can show that this norm is equivalent to the norm

$$
\|f\|_{r}=\|f\|_{\mathbf{E}}+\sum_{1 \leq i_{1}, \ldots, i_{r} \leq d}\left\|D_{i_{1}} \ldots D_{i_{k}} f\right\|_{\mathbf{E}}, \quad r \in \mathbb{N} .
$$

The mixed modulus of continuity is introduced as

$$
\begin{gathered}
\Omega^{r}(s, f)= \\
\sum_{1 \leq j_{1}, \ldots, j_{r} \leq d} \sup _{0 \leq \tau_{j_{1}} \leq s} \ldots \sup _{0 \leq \tau_{j_{r}} \leq s}\left\|\left(T_{j_{1}}\left(\tau_{j_{1}}\right)-I\right) \ldots\left(T_{j_{r}}\left(\tau_{j_{r}}\right)-I\right) f\right\|_{\mathbf{E}},
\end{gathered}
$$

where $f \in \mathbf{E}, r \in \mathbb{N}$, and $I$ is the identity operator in $\mathbf{E}$. Let $\mathcal{D}\left(D_{i}\right)$ be the domain of the operator $D_{i}$. For every $f \in \mathbf{E}$ we introduce a vector-valued function

$$
T f: \mathbb{R}^{d} \longmapsto \mathbf{E}
$$

defined as

$$
T f\left(t_{1}, t_{2}, \ldots, t_{d}\right)=T_{1}\left(t_{1}\right) T_{2}\left(t_{2}\right) \ldots T_{d}\left(t_{d}\right) f .
$$

Assumption 1. We assume that the following properties hold.

(1) There exists a set $\mathcal{G} \subset \mathbf{H}^{1}=\bigcap_{i=1}^{d} \mathcal{D}\left(D_{i}\right)$ which is dense in $\mathbf{H}$ and invariant with respect to all $T_{i}(t), 1 \leq i \leq d, t \geq 0$.

(2) For every $1 \leq i \leq d$, every $f \in \mathcal{G}$ and all $\mathbf{t}=\left(t_{1}, \ldots, t_{d}\right)$ in the standard open unit ball $U$ in $\mathbb{R}^{d}$

$$
D_{i} T f\left(t_{1}, \ldots, t_{d}\right)=\sum_{k=1}^{d} \zeta_{i}^{k}(\mathbf{t})\left(\partial_{k} T f\right)\left(t_{1}, \ldots, t_{d}\right),
$$

where $\zeta_{i}^{k}(\mathbf{t})$ belong to $C^{\infty}(U), \partial_{k}=\frac{\partial}{\partial t_{k}}$.

REMARK 1.1. Since $\mathbf{E}^{1}$ is invariant with respect to all bounded operators $T_{i}\left(t_{i}\right), \quad 1 \leq i \leq d$, we obtain for every $f \in \mathbf{E}^{1}$

$$
\begin{gathered}
T_{1}\left(t_{1}\right) T_{2}\left(t_{2}\right) \ldots D_{k} T_{k}\left(t_{k}\right) \ldots T_{d}\left(t_{d}\right) f= \\
T_{1}\left(t_{1}\right) T_{2}\left(t_{2}\right) \ldots T_{k-1}\left(t_{k-1}\right) \lim _{s \rightarrow 0} \frac{1}{s}\left(T_{k}\left(t_{k}+s\right)-T_{k}\left(t_{k}\right)\right) T_{k+1}\left(t_{k+1}\right) \ldots T_{d}\left(t_{d}\right) f=
\end{gathered}
$$




$$
\begin{gathered}
\lim _{s \rightarrow 0} \frac{1}{s} T_{1}\left(t_{1}\right) T_{2}\left(t_{2}\right) \ldots T_{k-1}\left(t_{k-1}\right)\left(T_{k}\left(t_{k}+s\right)-T_{k}\left(t_{k}\right)\right) T_{k+1}\left(t_{k+1}\right) \ldots T_{d}\left(t_{d}\right) f= \\
\frac{\partial}{\partial t_{k}} T_{1}\left(t_{1}\right) T_{2}\left(t_{2}\right) \ldots T_{d}\left(t_{d}\right) f=\left(\frac{\partial}{\partial t_{k}} T f\right)\left(t_{1}, \ldots, t_{d}\right) .
\end{gathered}
$$

Thus the formula (1.3) can be rewritten as

$$
D_{i} T_{1}\left(t_{1}\right) T_{2}\left(t_{2}\right) \ldots T_{d}\left(t_{d}\right) f=\sum_{k=1}^{d} \zeta_{i}^{k}(\mathbf{t}) T_{1}\left(t_{1}\right) T_{2}\left(t_{2}\right) \ldots D_{k} T_{k}\left(t_{k}\right) \ldots T_{d}\left(t_{d}\right) f
$$

where $\zeta_{i}^{k}(\mathbf{t})$ belong to $C^{\infty}(U), \mathbf{t}=\left(t_{1}, \ldots, t_{d}\right) \in U$.

REMARK 1.2. When semigroups commute with each other $T_{i}\left(t_{i}\right) T_{j}\left(t_{j}\right)=T_{j}\left(t_{j}\right) T_{i}\left(t_{i}\right), 1 \leq$ $i, j \leq d, t_{i}, t_{j} \geq 0$, then $\zeta_{i}^{k}=\delta_{i}^{k}$.

It is well known that most of remarkable properties of the so-called Besov functional spaces follow from the fact that they are interpolation spaces (see section 4 below) between two Sobolev spaces [3], [10]. For this reason we define Besov spaces by the formula

$$
\mathbf{E}^{\alpha, q}=\left(\mathbf{E}, \mathbf{E}^{r}\right)_{\alpha / r, q}^{K}, 0<\alpha<r \in \mathbb{N}, 1 \leq p, q \leq \infty,
$$

where $K$ is the so-called Peetre's interpolation functor (see section 4 below). The main result is the following.

THEOREM 1.3. If Assumption 1 is satisfied then the following holds true.

(1) The functionals $\Omega_{r}(s, f)$ and $K\left(s^{r}, f, \mathbf{E}, \mathbf{E}^{r}\right)$ are equivalent. Namely, there exist constants $c>0, C>0$, such that for all $f \in \mathbf{E}, s \geq 0$

(2) The norm of the Besov space $\mathbf{E}^{\alpha, q}=\left(\mathbf{E}, \mathbf{E}^{r}\right)_{\alpha / r, q}^{K}, 0<\alpha<r \in \mathbb{N}, 1 \leq$ $p, q \leq \infty$, is equivalent to the norm

$$
\|f\|_{\mathbf{E}}+\left(\int_{0}^{\infty}\left(s^{-\alpha} \Omega^{r}(s, f)\right)^{q} \frac{d s}{s}\right)^{1 / q}, 1 \leq q<\infty,
$$

with the usual modifications for $q=\infty$.

(3) The following isomorphism holds true $\left(\mathbf{E}, \mathbf{E}^{r}\right)_{\alpha / r, q}^{K}=\left(\mathbf{E}^{k_{1}}, \mathbf{E}^{k_{2}}\right)_{\left(\alpha-k_{1}\right) /\left(k_{2}-k_{1}\right), q}^{K}$, where $0 \leq k_{1}<\alpha<k_{2} \leq r \in \mathbb{N}, 1 \leq q \leq \infty$.

(4) If $\alpha$ is not integer then the norm (1.8) is equivalent to the norm

$$
\|f\|_{\mathbf{E}^{[\alpha]}}+\sum_{1 \leq j_{1}, \ldots, j_{[\alpha]} \leq d}\left(\int_{0}^{\infty}\left(s^{[\alpha]-\alpha} \Omega^{1}\left(s, D_{j_{1}} \ldots D_{j_{[\alpha]}} f\right)\right)^{q} \frac{d s}{s}\right)^{1 / q}
$$

where $[\alpha]$ is the integer part of $\alpha$.

(5) If $\alpha=k \in \mathbb{N}$ is an integer then the norm (1.8) is equivalent to the norm (Zygmund condition)

$$
\|f\|_{\mathbf{E}^{k-1}}+\sum_{1 \leq j_{1}, \ldots, j_{k-1} \leq d}\left(\int_{0}^{\infty}\left(s^{-1} \Omega^{2}\left(s, D_{j_{1}} \ldots D_{j_{k-1}} f\right)\right)^{q} \frac{d s}{s}\right)^{1 / q} .
$$

Next we make another assumption. 
Assumption 2. In addition to Assumption 1 we assume that $\mathbf{E}=\mathbf{H}$ is a Hilbert space and the following properties hold.

(1) The operator $L=D_{1}^{2}+\ldots+D_{d}^{2}$ is a non-negative self-adjoint operator in H.

(2) The domain $\mathcal{D}\left(L^{k / 2}\right), k \in \mathbb{N}$, of the non-negative square root $L^{k / 2}$ coincides with the space $\mathbf{H}^{k}$ and the norms (1.1) and $\|f\|_{\mathbf{H}}+\left\|L^{k / 2} f\right\|_{\mathbf{H}}$ are equivalent.

This assumption allows us to introduce notion of Paley-Wiener vectors (bandlimited vectors) (Definition 6.2) and to prove an abstract version of the PaleyWiener Theorem (Theorem 6.4).

To formulate an analog of the Shannon-type sampling in abstract Paley-Wiener spaces (Theorem 6.6) we consider the following assumptions.

Assumption 3. We assume that there exist $C, c>0$ and $m_{0} \geq 0$ such that for any $0<\rho<1$ there exists a set of functionals $\mathcal{A}^{(\rho)}=\left\{\mathcal{A}_{k}^{(\rho)}\right\}_{k \in \mathcal{K}}$, defined on $\mathbf{H}^{m_{0}}$, for which

$$
c \sum_{k}\left|\mathcal{A}_{k}^{(\rho)}(f)\right|^{2} \leq\|f\|_{\mathbf{H}}^{2} \leq C\left(\sum_{k \in \mathcal{K}}\left|\mathcal{A}_{k}^{(\rho)}(f)\right|^{2}+\rho^{2 m}\left\|L^{m / 2} f\right\|_{\mathbf{H}}^{2}\right),
$$

for all $f \in \mathbf{H}^{m}, m>m_{0}$.

This Abstract Sampling Theorem 6.6 is used to construct bandlimited frames in E (Theorem 6.7). By exploring relations between Interpolation and Approximation spaces we develop approximation theory by Paley-Wiener vectors in Theorems 7.5 and 7.6. Finally, in Theorem 7.7 we obtain description of Besov spaces in terms of frame coefficients.

In section 2 we show that our Assumptions 1 are satisfied for strongly continuous representations of Lie groups in Banach spaces and Assumptions 2 are satisfied for unitary representations of Lie groups in Hilbert spaces. Concerning Assumptions 3 we note that our results in [26]-33 imply that at least when one is considering a so-called regular or quasi-regular representation of a Lie group in a function space on a homogeneous manifold $M$, some specific sets of Dirac measures (or even more general functionals 29]) "uniformly" distributed over $M$ can serve as functionals $\mathcal{A}^{(\rho)}=\left\{\mathcal{A}_{k}^{(\rho)}\right\}_{k \in \mathcal{K}_{\rho}}$, where $\rho \in \mathbb{R}_{+}$represents a specific "spacing" of these Dirac measures.

It should be noted that due to importance of the theory of function spaces there is constant interest in extending classical constructions and results from Euclidean to non-Euclidean settings. It is impossible to list even the most significant publications on this subject which appeared during the last years. Here we mention just a very few papers which are relevant to our work [5], 15], [17.

\section{Lie groups and their representations}

2.1. Lie groups and their representations in Banach spaces. Lie algebra $\mathbf{g}$ of a Lie group $G$ can be identified with the tangent space $T_{e}(G)$ of $G$ at the identity $e \in G$. Let

$$
\exp (t X): T_{e}(G) \rightarrow G, t \in \mathbb{R}, X \in T_{e}(G),
$$


be the exponential geodesic map i. e. $\exp (t X)=\gamma(1)$, where $\gamma(t)$ is a geodesic of a fixed left-invariant metric on $G$ which starts at $e$ with the initial vector $t X \in T_{e}(G)$ : $\gamma(0)=e, \frac{d \gamma(0)}{d t}=t X$. It is known that exp is an analytic homomorphism of $\mathbb{R}$ onto one parameter subgroup $\exp t X$ of $G$ :

$$
\exp ((s+t) X)=\exp (s X) \exp (t X), s, t \in \mathbb{R} .
$$

Let $X_{1}, \ldots, X_{d}, d=\operatorname{dim} G$ form a basis in the Lie algebra of $G$, then one can consider the following coordinate system in a neighborhood of identity $e$

$$
\left(t_{1}, \ldots, t_{d}\right) \mapsto \exp \left(t_{1} X_{1}+\ldots+t_{d} X_{d}\right) .
$$

If $Y_{1}=s_{1} X_{1}+\ldots+s_{d} X_{d}$ and $Y_{2}=t_{1} X_{1}+\ldots+t_{d} X_{d}$ then

$$
\exp Y_{1} \exp Y_{2}=\exp Z \text {, }
$$

where $Z$ is given by the Campbell-Hausdorff formula

$Z=Y_{1}+Y_{2}+\frac{1}{2}\left[Y_{1}, Y_{2}\right]+\frac{1}{12}\left[Y_{1},\left[Y_{1}, Y_{2}\right]\right]-\frac{1}{12}\left[Y_{2},\left[Y_{1}, Y_{2}\right]\right]-\frac{1}{24}\left[Y_{2},\left[Y_{1},\left[Y_{1}, Y_{2}\right]\right]\right]+\ldots$.

It implies that $Z=\zeta_{1} X_{1}+\ldots+\zeta_{d} X_{d}$, where

$$
\zeta_{j}=s_{j}+t_{j}+O\left(\epsilon^{2}\right), \quad\left|t_{j}\right|,\left|s_{j}\right| \leq \epsilon, \quad 1 \leq j \leq d .
$$

One can also consider another local coordinate system around $e$ which is given by the formula

$$
\left(t_{1}, \ldots, t_{d}\right) \mapsto \varphi\left(\sum_{j=1}^{d} t_{j} X_{j}\right)=\exp \left(t_{1} X_{1}\right) \ldots \exp \left(t_{d} X_{d}\right) .
$$

Let us remind that a strongly continuous representation of a Lie group $G$ in a Banach space $\mathbf{E}$ is a homomorphism $g \mapsto T(g), g \in G, T(g) \in G L(\mathbf{E})$, of $G$ into the group $G L(\mathbf{E})$ of linear bounded invertible operators in $\mathbf{E}$ such that trajectory $T(g) f, g \in G, f \in \mathbf{E}$, is continuous with respect to $g$ for every $f \in \mathbf{E}$. We will consider only uniformly bounded representations. In this case one can introduce a new norm $\|f\|_{\mathbf{E}}^{\prime}=\sup _{g \in G}\|T(g) f\|_{\mathbf{E}}, \quad f \in \mathbf{E}$, in which $\|T(g) f\|_{\mathbf{E}}^{\prime} \leq\|f\|_{\mathbf{E}}^{\prime}$. Thus, without any restriction we will assume that the last inequality is satisfied in the original norm $\|\cdot\|_{\mathbf{E}}$. Let $X_{1}, \ldots, X_{d}$ be a basis in $\mathrm{g}$. With every $X_{j}, 1 \leq j \leq$ $d$, one associates a strongly continuous one-parameter group of isometries $t \mapsto$ $T\left(\exp t X_{j}\right), t \in \mathbb{R}$, whose generator is denoted as $D_{j}, 1 \leq j \leq d$.

LEMma 2.1. If $T: G \mapsto G L(\mathbf{E})$ is a strongly continuous bounded representation of $G$ in a Banach space $\mathbf{E}$ and $T_{j}(t)=T\left(\exp t X_{j}\right)$, where $\left\{X_{1}, \ldots, X_{d}\right\}$ is a basis in $\mathrm{g}$ then the Assumption 1 is satisfied for groups $T_{j}$ and their infinitesimal operators $D_{j}, 1 \leq j \leq d$.

Proof. The fact that the Garding space $\mathcal{G} \subset \mathbf{E}^{1}$ is dense in $\mathbf{E}$ and invariant is well known 13], 14. Since exp and $\varphi$ are diffeomorphisms in a neighborhood of zero in $\mathbf{g}$ the map $\exp ^{-1} \circ \varphi: \mathbf{g} \mapsto \mathbf{g}$ is also a diffeomorphism. The formulas (2.2) and (2.3) give connection between (2.1) and (2.4)

$$
\varphi\left(\sum_{j=1}^{d} t_{j} X_{j}\right)=\exp \left(\sum_{k=1}^{d} \alpha_{k}(t) X_{k}\right), t=\left(t_{1},,, t_{d}\right),
$$


where

$$
\alpha_{j}(t)=t_{j}+O\left(\epsilon^{2}\right), \quad\left|t_{j}\right| \leq \epsilon, \quad 1 \leq j \leq d .
$$

In particular, (2.2) implies

$$
\exp \tau X_{j} \exp \sum_{i=1}^{d} t_{i} X_{i}=\exp \sum_{k=1}^{d} \gamma_{k}^{j}(t, \tau) X_{k}, \quad t=\left(t_{1}, \ldots, t_{d}\right),
$$

where

$$
\gamma_{k}^{j}(t, \tau)=t_{k}+\tau \zeta_{k}^{j}(t)+\tau^{2} R_{k}^{j}(t, \tau),
$$

and $\zeta_{k}^{j}(t)=\delta_{k}^{j}+Q_{k}^{j}(t)$, where $\delta_{k}^{j}$ is the Kronecker symbol and $Q_{k}^{j}(t)$ and $R_{k}^{j}(t, \tau)$ are convergent series in $t_{1}, \ldots, t_{d}$ and $t_{1}, \ldots, t_{d}, \tau$ respectively.

Since for $f \in \mathbf{E}^{1}$ one has $D_{j} T(g) f=\left.\frac{d}{d \tau} T\left(\exp \tau X_{j}\right) T(g) f\right|_{\tau=0}$ we obtain for $f \in \mathbf{E}^{1}$ the following

$$
\begin{gathered}
D_{j} T_{1}\left(t_{1}\right) \ldots T_{d}\left(t_{d}\right) f=D_{j} T\left(\varphi\left(\sum_{i=1}^{d} t_{i} X_{i}\right)\right)=D_{j} T\left(\exp \sum_{i=1}^{d} \alpha_{i}(t) X_{i}\right) f= \\
\left.\frac{d}{d \tau} T\left(\exp \tau X_{j}\right) T\left(\varphi\left(\sum_{i=1}^{d} t_{i} X_{i}\right)\right) f\right|_{\tau=0}=\left.\frac{d}{d \tau} T\left(\exp \sum_{i=1}^{d} \gamma_{i}^{j}(\alpha, \tau) X_{i}\right) f\right|_{\tau=0},
\end{gathered}
$$

where $\alpha=\left(\alpha_{1}(t), \ldots, \alpha_{d}(t)\right)$ and according to (2.7) $\gamma_{i}^{j}(\alpha, \tau)=\alpha_{i}(t)+\tau \zeta_{i}^{j}(\alpha(t))+$ $\tau^{2} R_{i}^{j}(\alpha, \tau)$. By using the Chain Rule and (2.5) we finally obtain the formula (1.3)

$$
\begin{gathered}
D_{j} T_{1}\left(t_{1}\right) \ldots T_{d}\left(t_{d}\right) f=\left.\frac{d}{d \tau} T\left(\exp \sum_{i=1}^{d} \gamma_{i}^{j}(\alpha, \tau) X_{i}\right) f\right|_{\tau=0}= \\
\left.\sum_{k=1}^{d}\left(\left.\frac{d}{d \tau} \gamma_{k}^{j}(\alpha, \tau)\right|_{\tau=0}\right) \partial_{k} T\left(\exp \sum_{i=1}^{d} \gamma_{i}^{j}(\alpha, \tau) X_{i}\right) f\right|_{\tau=0}=\sum_{k=1}^{d} \zeta_{k}^{j}(t) \partial_{k} T_{1}\left(t_{1}\right) \ldots T_{d}\left(t_{d}\right) f .
\end{gathered}
$$

Lemma is proved.

2.2. Unitary representations in Hilbert spaces. A strongly continuous unitary representation of a Lie group $G$ in a Hilbert space $\mathbf{H}$ is a homomorphism $T: G \mapsto U(\mathbf{H})$ where $U(\mathbf{E})$ is the group of unitary operators of $\mathbf{H}$ such that $T(g) f, g \in G$, is continuous on $G$ for any $f \in \mathbf{H}$. The Garding space $\mathcal{G}$ is defined as the set of vectors $h$ in $\mathbf{H}$ that have the representation $h=\int_{G} \varphi(g) T(g) f d g$, where $f \in \mathbf{H}, \quad \varphi \in C_{0}^{\infty}(G), \quad d g$ is a left-invariant measure on $G$. If $X \in \mathbf{g}$ is identified with a right-invariant vector field

$$
X \varphi(g)=\lim _{t \rightarrow 0} \frac{\varphi(\exp t X \cdot g)-\varphi(g)}{t},
$$

then one has a representation $D(X)$ of $\mathbf{g}$ by operators which act on $\mathcal{G}$ by the formula $D(X) h=-\int_{G} X \varphi(g) T(g) f d g$. It is known that $\mathcal{G} \subset \bigcap_{r \in N} \mathbf{H}^{r}=\mathbf{H}^{\infty}$ is invariant with respect to all operators $D(X), X \in \mathbf{g}$, and dense in every $\mathbf{H}^{r}$. If $X_{1}, \ldots, X_{d}$ is a basis in $\mathbf{g}$ and $D_{i}=D\left(X_{i}\right), 1 \leq i \leq d$, we consider the operator $L_{\mathcal{G}}=-\sum_{i=1}^{d} D_{i}^{2}$ defined on $\mathcal{G}$.

Since $L_{\mathcal{G}}$ is symmetric and the differential operator $-\sum_{i=1}^{d} X_{i}^{2}$ is elliptic on the group $G$ the Theorem 2.2 in $\mathbf{1 4}$ implies that $L_{\mathcal{G}}$ is essentially self-adjoint, which means $\bar{L}_{\mathcal{G}}=L_{\mathcal{G}}^{*}$. In other words, the closure $\bar{L}_{\mathcal{G}}=L$ of $L_{\mathcal{G}}$ from $\mathcal{G}$ is a self-adjoint operator. Obviously, $L \geq 0$. We introduce the self-adjoint operator $\Lambda=I+L \geq 0$. 
THEOREM 2.2. The space $\mathbf{H}^{r}$ with the norm (1.1) is isomorphic to the domain of $\Lambda^{r / 2}$ with the norm $\left\|\Lambda^{r / 2} f\right\|_{\mathbf{H}}$.

PRoOF. In the case $r=2 k$, the inequality

$$
\|f\|_{\mathbf{H}^{2 k}} \leq C(k)\left\|\Lambda^{k} f\right\|_{\mathbf{H}}
$$

is shown in 13, Lemma 6.3. The reverse inequality is obvious. We consider now the case $r=2 k+1$. If $f \in \mathbf{H}^{2}=\mathcal{D}(\Lambda)$, then since $\mathcal{D}(\Lambda) \subset \mathcal{D}\left(\Lambda^{1 / 2}\right)$ we have

$$
\begin{gathered}
\|f\|_{\mathbf{H}}^{2}+\sum_{j}\left\|D_{j} f\right\|_{\mathbf{H}}^{2}=\langle f, f\rangle+\sum_{j}\left\langle D_{j} f, D_{j} f\right\rangle=\langle f, f\rangle+\left\langle-\sum_{j} D_{j}^{2} f, f\right\rangle= \\
\left\langle f-\sum_{j} D_{j}^{2} f, f\right\rangle=\langle\Lambda f, f\rangle=\left\|\Lambda^{1 / 2} f\right\|_{\mathbf{H}}^{2} .
\end{gathered}
$$

These equalities imply that $\mathbf{H}^{1}$ is isomorphic to $\mathcal{D}\left(\Lambda^{1 / 2}\right)$. Our goal is to to prove existence of an isomorphism between $\mathbf{H}^{2 k+1}$ and $\mathcal{D}\left(\Lambda^{k+1 / 2}\right)$. It is enough to establish equivalence of the corresponding norms on the set $\mathbf{H}^{4 k+2}=\mathcal{D}\left(\Lambda^{2 k+1}\right)$ since the latest is dense in $\mathbf{H}^{2 k+1}$. If $f \in \mathbf{H}^{4 k+2} \subset \mathbf{H}^{2 k}$ then $D_{j} f \in \mathbf{H}^{4 k+1} \subset \mathbf{H}^{2 k}$ and $\Lambda^{k} f=\sum_{m \leq k} \sum D_{j_{1}}^{2} \ldots D_{j_{m}}^{2} f$. Thus if $f \in \mathbf{H}^{4 k+2}$ then

$$
\left\|D_{j_{1}} \ldots D_{j_{2 k+1}} f\right\|_{\mathbf{H}} \leq C\left\|\Lambda^{k} D_{j_{2 k+1}} f\right\|_{\mathbf{H}}=\left\|\sum_{m \leq k} \sum D_{j_{1}}^{2} \ldots D_{j_{m}}^{2} D_{j_{2 k+1}} f\right\|_{\mathbf{H}} .
$$

Multiple applications of the identity $D_{i} D_{j}-D_{j} D_{i}=\sum_{k} c_{i, j}^{k} D_{k}$ which holds on $\mathbf{H}^{2}$

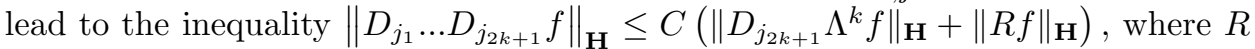
is a polynomial in $D_{1}, \ldots, D_{d}$ whose degree $\leq 2 k$. According to (2.8) and (2.2) we have that

$$
\left\|D_{j_{2 k+1}} \Lambda^{k} f\right\|_{\mathbf{H}} \leq\left\|\Lambda^{1 / 2} \Lambda^{k} f\right\|_{\mathbf{H}}=\left\|\Lambda^{k+1 / 2} f\right\|_{\mathbf{H}}
$$

and also $\|R f\|_{\mathbf{H}} \leq\|f\|_{\mathbf{H}^{2 k}} \leq C(k)\left\|\Lambda^{k} f\right\|_{\mathbf{H}}$. Since $\left\|\Lambda^{k} f\right\|_{\mathbf{H}}$ is not decreasing with $k$ we get the following estimate

$$
\left\|D_{j_{1}} \ldots D_{j_{2 k+1}} f\right\|_{\mathbf{H}} \leq C(k)\left\|\Lambda^{k+1 / 2} f\right\|_{\mathbf{H}}, \quad f \in \mathbf{H}^{4 k+2} .
$$

Now, since for $f \in \mathbf{H}^{4 k+2}$ we have $D_{j_{1}} \ldots D_{j_{2 k}} f \in \mathbf{H}^{2 k+2} \subset \mathbf{H}^{1}=\mathcal{D}\left(\Lambda^{1 / 2}\right)$, and the equality $\Lambda^{k} f=\sum_{m \leq k} \sum D_{j_{1}}^{2} \ldots D_{j_{m}}^{2} f$, holds we obtain, by using (2.2)

$$
\left\|\Lambda^{k+1 / 2} f\right\|_{\mathbf{H}}=\left\|\Lambda^{1 / 2} \sum_{m \leq k} \sum D_{j_{1}}^{2} \ldots D_{j_{m}}^{2} f\right\|_{\mathbf{H}} \leq C\|f\|_{\mathbf{H}^{2 k+1}}, \quad C=C(k) .
$$

Theorem is proved.

COROLlaRY 2.1. If $T$ is a strongly continuous unitary representation of a Lie group in a Hilbert space $\mathbf{H}$ and $X_{1}, \ldots, X_{d}$ is a basis in the corresponding algebra Lie $\mathbf{g}$ then for $T_{j}(t)=T\left(\exp t X_{j}\right), 1 \leq j \leq d$, and their generators $D_{j}, 1 \leq j \leq d$, the Assumption 2 is satisfied. 


\section{Hardy-Steklov operator associated with operators $D_{1}, D_{2}, \ldots, D_{d}$.}

We return to the general set up. Namely, we consider a Banach space $\mathbf{E}$ and operators $D_{1}, D_{2}, \ldots, D_{d}$ which generate strongly continuous uniformly bounded semigroups $T_{1}(t), T_{2}(t), \ldots, T_{d}(t),\|T(t)\| \leq 1, t \geq 0$. It is assumed that the Assumption 1 holds.

3.1. Preliminaries. If $D$ generates in $\mathbf{E}$ a strongly continuous bounded semigroup $T_{D}(t), t \geq 0$, and

$$
\Omega^{r}(s, f)=\sup _{0 \leq \tau \leq s}\left\|\left(T_{D}(\tau)-I\right)^{r} f\right\|_{\mathbf{E}}
$$

where $I$ is the identity operator, then one can easily prove the following two inequalities

$$
\Omega^{m}(f, s) \leq s^{k} \Omega^{m-k}\left(D^{k} f, s\right),
$$

and

$$
\Omega^{m}(f, a s) \leq(1+a)^{m} \Omega^{m}(f, s), a \in \mathbb{R} .
$$

Let $F\left(x_{1}, x_{2}, \ldots, x_{N}\right)$ be a function on $\mathbb{R}^{N}$ that takes values in the Banach space $\mathbf{E}$

$$
F: \mathbb{R}^{N} \longmapsto \mathbf{E} .
$$

For $1 \leq i \leq N$ we introduce difference operator by the formula

$$
\begin{gathered}
\left(\Delta_{i}(s) F\right)\left(x_{1}, x_{2}, \ldots, x_{N}\right)=F\left(x_{1}, x_{2}, \ldots, x_{i-1}, s, x_{i+1}, \ldots, x_{N}\right)- \\
F\left(x_{1}, x_{2}, \ldots, x_{i-1}, 0, x_{i+1}, \ldots, x_{N}\right) .
\end{gathered}
$$

For a scalar differentiable function $\theta: \mathbb{R}^{N} \longmapsto \mathbb{R}$ and for $1 \leq i_{1}, \ldots, i_{l} \leq N, i_{m} \neq i_{k}$, the following inequality holds

$$
\max _{0 \leq x_{j} \leq s_{j}}\left|\Delta_{i_{1}}\left(s_{i_{1}}\right) \ldots \Delta_{i_{l}}\left(s_{i_{l}}\right) \theta\left(x_{1}, \ldots, x_{N}\right)\right| \leq s_{i_{1}} \ldots s_{i_{l}} \max _{0 \leq x_{j} \leq s_{j}}\left|\frac{\partial^{k}}{\partial_{i_{1}} \ldots \partial_{i_{k}}} \theta\left(x_{1}, \ldots, x_{N}\right)\right| .
$$

One has for $1 \leq i \leq N$

$$
\begin{gathered}
\Delta_{i}(s)(\theta F)\left(x_{1}, \ldots, x_{N}\right)=\Delta_{i}(s) \theta\left(x_{1}, \ldots, x_{N}\right) F\left(x_{1}, \ldots, x_{i-1}, s, x_{i+1}, \ldots, x_{N}\right)+ \\
\theta\left(x_{1}, \ldots, x_{i-1}, 0, x_{i+1}, \ldots, x_{N}\right) \Delta_{i}(s) F\left(x_{1}, \ldots, x_{N}\right)
\end{gathered}
$$

and then if $i_{m} \neq i_{k}$ one has

$$
\Delta_{i_{1}}\left(s_{i_{1}}\right) \ldots \Delta_{i_{l}}\left(s_{i_{l}}\right)(\theta F)\left(x_{1}, \ldots, x_{N}\right)=
$$

$$
\sum \Delta_{i_{1}^{(1)}}\left(s_{i_{1}^{(1)}}\right) \ldots \Delta_{i_{l}^{(1)}}\left(s_{i_{l}^{(1)}}\right) \theta\left(x_{i_{1}^{(2)}, \ldots, i_{l}^{(2)}}^{(0)}\right) \Delta_{i_{1}^{(2)}}\left(s_{i_{1}^{(2)}}\right) \ldots \Delta_{i_{l}^{(2)}}\left(s_{i_{l}^{(2)}}\right) F\left(x_{i_{1}^{(1)}, \ldots, i_{l}^{(1)}}^{(s)}\right),
$$

where the sum is taken over all possible partitions of the natural vector $\left(i_{1}, \ldots, i_{l}\right)$ in the sum of nonnegative integer vectors $\left(i_{1}^{(1)}, \ldots, i_{l}^{(1)}\right)$ and $\left(i_{1}^{(2)}, \ldots, i_{l}^{(2)}\right)$. The $x_{i_{1}^{(2)}, \ldots, i_{l}^{(2)}}^{(0)}$ denotes a vector obtained from the vector $\left(x_{1}, \ldots, x_{N}\right)$ by replacing the coordinates $x_{i_{1}^{(2)}, \ldots, i_{l}^{(2)}}$ by 0 and $x_{i_{1}^{(1)}, \ldots, i_{l}^{(1)}}^{(s)}$, denotes the vector obtained by replacing $i_{1}^{(1)}, \ldots, i_{l}^{(1)}$ by $s_{i_{1}^{(1)}}, \ldots, s_{i_{l}^{(1)}}$, respectively.

If $F$ is a differentiable function and $m \neq i$ then we have

$$
\Delta_{i}(s) \frac{\partial}{\partial x_{m}} F\left(x_{1}, x_{2}, \ldots, x_{N}\right)=\frac{\partial}{\partial x_{m}} \Delta_{i}(s) F\left(x_{1}, x_{2}, \ldots, x_{N}\right), \quad m \neq i .
$$


In particular, for any $r \geq 2$ for $\tau_{j}=\tau_{j, 1}+\ldots+\tau_{j, r}, j=1, \ldots, d, 1 \leq i, i^{\prime} \leq d, 1 \leq$ $k, k^{\prime} \leq r$, if $\left(i^{\prime}, k^{\prime}\right) \neq(i, k)$ we have

$$
\frac{\partial}{\partial \tau_{i^{\prime}, k^{\prime}}} \Delta_{i, k}(s) \prod_{j=1}^{d} T_{j}\left(\tau_{j}\right) f=\Delta_{i, k}(s) \frac{\partial}{\partial \tau_{i^{\prime}, k^{\prime}}} \prod_{j=1}^{d} T_{j}\left(\tau_{j}\right) f, \quad f \in \mathbf{E}^{1},
$$

or, equvalently,

$$
\frac{\partial}{\partial \tau_{i^{\prime}}} \Delta_{i, k}(s) \prod_{j=1}^{d} T_{j}\left(\tau_{j}\right) f=\Delta_{i, k}(s) \frac{\partial}{\partial \tau_{i^{\prime}}} \prod_{j=1}^{d} T_{j}\left(\tau_{j}\right) f, \quad f \in \mathbf{E}^{1},
$$

where the right-hand side and $\Delta_{i, k}(s) \prod_{j=1}^{d} T_{j}\left(\tau_{j}\right) f$ are defined according to (3.1). In the same notations $\tau_{j}=\tau_{j, 1}+\ldots+\tau_{j, r}, 1 \leq m, i \leq d, 1 \leq k \leq r$, we clearly have the identity

$$
D_{m} \Delta_{i, k}(s) \prod_{j=1}^{d} T_{j}\left(\tau_{j}\right) f=\Delta_{i, k}(s) D_{m} \prod_{j=1}^{d} T_{j}\left(\tau_{j}\right) f, \quad f \in \mathbf{E}^{1},
$$

which implies for every $f \in \mathbf{E}^{1}$ the next formula

$$
D_{m} \Delta_{i_{1}, k_{1}}(s) . . \Delta_{i_{l}, k_{l}}(s) \prod_{j=1}^{d} T_{j}\left(\tau_{j}\right) f=\Delta_{i_{1}, k_{1}}(s) . . \Delta_{i_{l}, k_{l}}(s) D_{m} \prod_{j=1}^{d} T_{j}\left(\tau_{j}\right) f .
$$

Using (3.7), (1.3), (3.1) and (3.6) we obtain for $f \in \mathbf{E}^{1}, \tau_{j}=\tau_{j, 1}+\ldots+\tau_{j, r}$, $j=1, \ldots, d, r \geq l+1,1 \leq m \leq d$,

$$
D_{m} \Delta_{i_{1}, k_{1}}(s) \ldots \Delta_{i_{l}, k_{l}}(s) \prod_{j=1}^{d} T_{j}\left(\tau_{j}\right) f=
$$

$\sum \sum_{i_{l+1}=1}^{d} \Delta_{i_{1}^{(1)}, k_{1}^{(1)}}(s) \ldots \Delta_{i_{l}^{(1)}, k_{l}^{(1)}}(s) \zeta_{m}^{i_{l+1}}(\ldots) \frac{\partial}{\partial \tau_{i_{l+1}}} \Delta_{i_{1}^{(2)}, k_{1}^{(2)}}(s) \ldots \Delta_{i_{l}^{(2)}, k_{l}^{(2)}}(s) \prod_{j=1}^{d} T_{j}(\ldots) f$

where outer summation $\sum$ and arguments of $\zeta_{m}^{i_{l+1}}(\ldots)$ and $\prod_{j=1}^{d} T_{j}(\ldots) f$ the same as in (3.1).

3.2. The Hardy-Steklov operator. We introduce a generalization of the classical Hardy-Steklov operator. For a positive small $s$, natural $r$ and $1 \leq j \leq d$ we set

$$
H_{j . r}(s) f=(s / r)^{-r} \int_{0}^{s / r} \ldots \int_{0}^{s / r} \sum_{k=1}^{r}(-1)^{k} C_{r}^{k} T_{j}\left(k\left(\tau_{j, 1}+\ldots+\tau_{j, r}\right) f d \tau_{j, 1} \ldots d \tau_{j, r},\right.
$$

where $C_{r}^{k}$ are the binomial coefficients and then define the Hardy-Steklov operator: $H_{r}(s) f=\prod_{j=1}^{d} H_{j, r}(s) f=H_{1, r}(s) H_{2, r}(s) \ldots H_{d, r}(s) f, f \in \mathbf{E}$. For every fixed $f \in \mathbf{E}$ the function $H_{r}(s) f$ is an abstract valued function from $\mathbb{R}$ to $\mathbf{E}$ and it is a linear combination of some abstract valued functions of the form

$$
(s / r)^{-r d} \int_{0}^{s / r} \ldots \int_{0}^{s / r} T f(\tau) d \tau_{1,1} \ldots d \tau_{d, r}
$$


where

$$
\begin{gathered}
\tau=\left(k_{1} \tau_{1}, k_{2} \tau_{2}, \ldots, k_{d} \tau_{d}\right), \quad 1 \leq k_{j} \leq r, \\
\tau_{j}=\left(\tau_{j, 1}+\tau_{j, 2}+\ldots+\tau_{j, r}\right), \quad 1 \leq j \leq d,
\end{gathered}
$$

and

$$
T f(\tau)=T_{1}\left(k_{1} \tau_{1}\right) T_{2}\left(k_{2} \tau_{2}\right) \ldots T_{d}\left(k_{d} \tau_{d}\right) f
$$

LEMma 3.1. The following holds:

(1) For every $f \in \mathbf{E}$ the function $H_{r}(s) f$ maps $\mathbb{R}$ to $\mathbf{E}^{r}$.

(2) For every $0 \leq q \leq r$ the "mixed derivative" $D_{j_{1}} \ldots D_{j_{q}} H_{r}(s) f, \quad 1 \leq j_{k} \leq d$ is another abstract valued function with values in $\mathbf{E}^{r-q}$ and it is a linear combination of abstract valued functions (with values in $\mathbf{E}^{r}$ ) of the form

$$
(s / r)^{-r d} \underbrace{\int_{0}^{s / r} \cdots . . \int_{0}^{s / r}}_{r d-m} \mu_{j_{1}, \ldots, j_{q}}^{i_{1}, \ldots, i_{l} ; m}(\cdot) \Delta_{i_{1}, k_{1}}(s / r) \ldots . \Delta_{i_{l}, k_{l}}(s / r) T f(\cdot) d \cdot
$$

where

$$
\max _{0 \leq \tau_{i, j} \leq s}\left|\partial^{p} \mu_{j_{1}, \ldots, j_{q}}^{i_{1}, \ldots, i_{i} ; m}(\cdot)\right| \leq c s^{m-l}, \quad p \in \mathbb{N} \cup\{0\},
$$

and $0<s<1,0 \leq m \leq r d, 0 \leq l \leq m$, where $l=0$ corresponds to the case when the set of indices $\left\{i_{1}, \ldots, i_{l}\right\}$ is empty.

Proof. The proof proceeds by induction on $q$. We shall show that $H_{r}(s) f$ is in $\mathbf{E}^{1}$ for every $f \in \mathbf{E}$. Let's assume first that $f \in \mathbf{E}^{1}$. Since every $D_{j}, 1 \leq j \leq d$, is a closed operator to show that the term (3.9) belongs to $\mathcal{D}\left(D_{j}\right)$ it is sufficient to show existence of the integral (see notations (3.10), (3.11)

$$
\left(\frac{s}{r}\right)^{-r d} \int_{0}^{s / r} \ldots \int_{0}^{s / r} D_{j} T f(\tau) d \tau_{1,1} \ldots d \tau_{d, r} .
$$

According to (1.3) the last integral equals to

$$
\sum_{i=1}^{d}\left(\frac{s}{r}\right)^{-r d} \int_{0}^{s / r} \ldots \int_{0}^{s / r} \zeta_{j}^{i}(\tau) \partial_{i} T f\left(k_{1} \tau_{1}, \ldots, k_{d} \tau_{d}\right) d \tau_{1,1} \ldots d \tau_{d, r},
$$

where $\tau$ and $\tau_{j}$ described in (3.10) and (3.11). Since derivative $\partial_{i}$ is the same as the derivative $\frac{\partial}{\partial \tau_{i_{1}, 1}}$ the integration by parts formula and (3.1) allow to continue (3.16) as follows

$$
\begin{aligned}
& (s / r)^{-r d} \sum_{i=1}^{d}(\underbrace{\int_{0}^{s / r} \ldots \int_{0}^{s / r}}_{r d-1} \zeta_{j}^{i}\left(\tau_{i}^{(s / r)}\right) \Delta_{i}(s / r) T f(\tau)(d \tau)_{i})+ \\
& (s / r)^{-r d} \sum_{i=1}^{d}(\underbrace{\int_{0}^{s / r} \ldots \int_{0}^{s / r}}_{r d-1}\left(\Delta_{i}(s / r) \zeta_{j}^{i}(\tau)\right) T f\left(\tau_{i}^{(0)}\right)(d \tau)_{i})-
\end{aligned}
$$




$$
(s / r)^{-r d} \sum_{i=1}^{d}(\underbrace{\int_{0}^{s / r} \ldots \int_{0}^{s / r}}_{r d}\left(\partial_{i} \zeta_{j}^{i}\right)(\tau) T f(\tau) d \tau)=B_{j}(s) f,
$$

where $\tau=\left(\tau_{1,1}+\ldots+\tau_{1, r}, \ldots, \tau_{n, 1}+\ldots+\tau_{n, r}\right), \tau_{i}^{s / r}=\left(\tau_{1,1}+\ldots+\tau_{1, r}, \ldots, \tau_{i, 1}+\ldots+\right.$ $\left.\tau_{i, r-1}+s / r, \ldots, \tau_{n, 1}+\ldots+\tau_{n, r}\right), \tau_{i}^{0}=\left(\tau_{1,1}+\ldots+\tau_{1, r}, \ldots, \tau_{i, 1}+\ldots+\tau_{i, r-1}+0, \ldots, \tau_{n, 1}+\right.$ $\left.\ldots+\tau_{n, r}\right), \quad d \tau=d \tau_{1,1} \ldots d \tau_{n, r}$, and $(d \tau)_{i}=d \tau_{1,1} \ldots d \tau_{i, r-1} \widehat{d \tau_{i, r}} d \tau_{i+1,1} \ldots d \tau_{n, r}$, where the term $d \tau_{i, r}$ is missing.

Since integrand of each of the three integrals is bounded it implies existence of (3.15) which, in turn, shows that (3.9) is an element of $\mathcal{D}\left(D_{j}\right)$ for every $1 \leq j \leq d$.

Thus if $f$ belongs to $\mathbf{E}^{1}$ then $H_{r}(s) f$ takes values in $\mathbf{E}^{1}$ and the formula $D_{j} H_{r}(s) f=B_{j}(s) f$ holds where the operator $B_{m}(s)$ is bounded. This fact along with the fact that $\mathbf{E}^{1}$ is dense in $\mathbf{E}$ implies the formula $D_{j} H_{r}(s) f=B_{j}(s) f$ for every $f \in \mathbf{E}$. Thus we proved the first part of the Lemma for $q=1$.

To verify the second claim of the Lemma it is sufficient to note that in the first line of (3.2) one has $m=l=q=1, j=j_{1}, \mu_{j_{1}}^{1,1}=\zeta_{j}^{i}$, in the second line $m=1, l=$ $0, q=1, j=j_{1}, \mu_{j_{1}}^{0,1}=\Delta_{i}(s / r) \zeta_{j}^{i}$, in the third line $m=l=0, q=1, \mu_{j_{1}}^{0,0}=\partial_{i} \zeta_{j}^{i}$. It is easy to verify that in all of these cases the estimates (3.14) hold.

We now assume that theorem is proved for all $q<r$ and we shall prove it for $q+1 \leq r$. Suppose $f \in \mathbf{E}^{1}$. We consider

$$
\int_{0}^{s / r} \ldots . . \int_{0}^{s / r} \mu_{j_{1}, \ldots, j_{q}}^{i_{1}, \ldots, i_{l} ; m}(\cdot) D_{j_{q+1}} \Delta_{i_{1}, k_{1}}(s / r) \ldots . \Delta_{i_{l}, k_{l}}(s / r) T(\cdot) f d \cdot .
$$

By (3.1) this expression is decomposed into a sum of terms of the form

$$
\int_{0}^{s / r} \cdots \int_{0}^{s / r} \nu_{j_{1}, \ldots, j_{q+1}}^{i_{1}, \ldots, i_{l+1} ; m}(\cdot) \partial_{i_{l+1}} \Delta_{i_{1}^{(2)}, k_{1}^{(2)}}(s / r) \ldots \Delta_{i_{l}^{(2)}, k_{l}^{(2)}}(s / r) T(\tau) f d \tau,
$$

where $\nu_{j_{1}, \ldots, j_{q+1}}^{i_{1}, \ldots, i_{l+1} ; m}$ are constructed from $\mu_{j_{1}, \ldots, j_{q}}^{i_{1}, \ldots, i_{i} ; m}$ in accordance with (3.1). Integrating by parts as in the first step of induction, we obtain that the integral (3.19) exists and is equal to the value of some bounded operator at an element $f \in \mathbf{E}^{1}$. As a result we obtain that $H_{r}(s)$ belongs to the space $\mathbf{E}^{q+1}$ and also that $D_{j_{1}} \ldots D_{j_{q+1}} H_{r}(s) f$ is a linear combination of terms of the form (3.13) . The rest of the theorem fellows from the induction hypothesis. Theorem is proved.

\section{Interpolation spaces}

The goal of the section is to introduce basic notions of the theory of interpolation spaces [1, 3], 10]. Later in section 7 we will also introduce the so-called approximation spaces [18, 4]. It is important to realize that the relations between interpolation and approximation spaces cannot be described in the language of normed spaces. We have to make use of quasi-normed linear spaces in order to treat them simultaneously.

A quasi-norm $\|\cdot\|_{\mathbf{E}}$ on linear space $\mathbf{E}$ is a real-valued function on $\mathbf{E}$ such that for any $f, f_{1}, f_{2} \in \mathbf{E}$ the following holds true: (1) $\|f\|_{\mathbf{E}} \geq 0 ; \quad(2)\|f\|_{\mathbf{E}}=0 \Longleftrightarrow f=0$; (3) $\|-f\|_{\mathbf{E}}=\|f\|_{\mathbf{E}} ; \quad$ (4) there exists some $C_{\mathbf{E}} \geq 1$ such that $\left\|f_{1}+f_{2}\right\|_{\mathbf{E}} \leq$ $C_{\mathbf{E}}\left(\left\|f_{1}\right\|_{\mathbf{E}}+\left\|f_{2}\right\|_{\mathbf{E}}\right)$. Two quasi-normed linear spaces $\mathbf{E}$ and $\mathbf{F}$ form a pair if they are linear subspaces of a common linear space $\mathbf{A}$ and the conditions $\left\|f_{k}-g\right\|_{\mathbf{E}} \rightarrow 0$, 
and $\left\|f_{k}-h\right\|_{\mathbf{F}} \rightarrow 0$ imply equality $g=h$ (in $\mathbf{A}$ ). For any such pair $\mathbf{E}, \mathbf{F}$ one can construct the space $\mathbf{E} \cap \mathbf{F}$ with quasi-norm $\|f\|_{\mathbf{E} \cap \mathbf{F}}=\max \left(\|f\|_{\mathbf{E}},\|f\|_{\mathbf{F}}\right)$ and the sum of the spaces, $\mathbf{E}+\mathbf{F}$ consisting of all sums $f_{0}+f_{1}$ with $f_{0} \in \mathbf{E}, f_{1} \in \mathbf{F}$, and endowed with the quasi-norm $\|f\|_{\mathbf{E}+\mathbf{F}}=\inf _{f=f_{0}+f_{1}, f_{0} \in \mathbf{E}, f_{1} \in \mathbf{F}}\left(\left\|f_{0}\right\|_{\mathbf{E}}+\left\|f_{1}\right\|_{\mathbf{F}}\right)$.

Quasi-normed spaces $\mathbf{H}$ with $\mathbf{E} \cap \mathbf{F} \subset \mathbf{H} \subset \mathbf{E}+\mathbf{F}$ are called intermediate between $\mathbf{E}$ and $\mathbf{F}$. If both $E$ and $F$ are complete the inclusion mappings are automatically continuous. An additive homomorphism $T: \mathbf{E} \rightarrow \mathbf{F}$ is called bounded if $\|T\|=\sup _{f \in \mathbf{E}, f \neq 0}\|T f\|_{\mathbf{F}} /\|f\|_{\mathbf{E}}<\infty$. An intermediate quasi-normed linear space $\mathbf{H}$ interpolates between $\mathbf{E}$ and $\mathbf{F}$ if every bounded homomorphism $T: \mathbf{E}+\mathbf{F} \rightarrow \mathbf{E}+\mathbf{F}$ which is a bounded homomorphism of $\mathbf{E}$ into $\mathbf{E}$ and a bounded homomorphism of $\mathbf{F}$ into $\mathbf{F}$ is also a bounded homomorphism of $\mathbf{H}$ into $\mathbf{H}$. On $\mathbf{E}+\mathbf{F}$ one considers the so-called Peetre's $K$-functional

$$
K(f, t)=K(f, t, \mathbf{E}, \mathbf{F})=\inf _{f=f_{0}+f_{1}, f_{0} \in \mathbf{E}, f_{1} \in \mathbf{F}}\left(\left\|f_{0}\right\|_{\mathbf{E}}+t\left\|f_{1}\right\|_{\mathbf{F}}\right) .
$$

The quasi-normed linear space $(\mathbf{E}, \mathbf{F})_{\theta, q}^{K}$, with parameters $0<\theta<1,0<q \leq \infty$, or $0 \leq \theta \leq 1, q=\infty$, is introduced as the set of elements $f$ in $\mathbf{E}+\mathbf{F}$ for which

$$
\|f\|_{\theta, q}=\left(\int_{0}^{\infty}\left(t^{-\theta} K(f, t)\right)^{q} \frac{d t}{t}\right)^{1 / q}<\infty .
$$

It turns out that $(\mathbf{E}, \mathbf{F})_{\theta, q}^{K}$ with the quasi-norm (4.1) interpolates between $\mathbf{E}$ and $\mathbf{F}$.

\section{Approximation by Hardy-Steklov averages, K-functor and modulus of continuity}

We are going to prove items (1) and (2) of Theorem 1.3

Proof. First we prove the right-hand side of the inequality (1.7). The following simple lemma plays an important role in the roof.

LEMMA 5.1. In any ring $\mathcal{R}$ with multiplicative identity 1 the following formulas hold for $a_{1}, a_{2}, \ldots ., a_{n} \in \mathcal{R}$,

$$
\begin{gathered}
a_{1} a_{2} \ldots a_{n}-1=a_{1}\left(a_{2}-1\right)+\ldots+a_{1} a_{2} \ldots a_{n-1}\left(a_{n}-1\right), \\
\left(a_{1}-1\right) a_{2} \ldots a_{n}=\left(a_{1}-1\right)+a_{1}\left(a_{2}-1\right)+\ldots+a_{1} a_{2} \ldots a_{n-1}\left(a_{n}-1\right) .
\end{gathered}
$$

By using the first formula we obtain

$$
\begin{gathered}
\left\|(-1)^{n(r+1)} H_{r}(s) f-f\right\|_{\mathbf{E}}=\left\|\prod_{j=1}^{n}(-1)^{n+1} H_{j, r}(s) f-f\right\|_{\mathbf{E}} \leq \\
c \sum_{j=1}^{n} \sup _{0 \leq \tau_{j} \leq s / r}\left\|\left(T_{j}\left(\tau_{j}\right)-I\right)^{r} f\right\|_{\mathbf{E}} \leq C \Omega^{r}(s, f) .
\end{gathered}
$$

To estimate $s^{r}\left\|H_{r}(s) f\right\|_{\mathbf{E}^{r}}$ we note that $\left\|H_{r}(s) f\right\|_{\mathbf{E}} \leq C\|f\|_{\mathbf{E}}$. According to Lemma 3.1 the quantity $\left\|D_{j_{1}} \ldots D_{j_{r}} H_{r}(s) f\right\|_{\mathbf{E}}$ is estimated for $0 \leq s \leq 1$ by

$$
s^{-l} \sup _{0 \leq \tau_{j, k} \leq s}\left\|\Delta_{j_{1}, k_{1}}(s / r) \ldots \Delta_{j_{l}, k_{l}}(s / r) T(\cdot) f\right\|_{\mathbf{E}},
$$

where $T(\cdot)=\prod_{j=1}^{n} \prod_{k=1}^{r} T_{j}\left(\tau_{j, k}\right)$. By the definition of $\Delta_{j, k}(s / r)$ the expression $\Delta_{j, k}(s / r) T(\cdot)$ differs from $T(\cdot)$ only in that in place of the factor $T_{j}\left(\tau_{j, k}\right)$ the factor 
$T_{j}(s / r)-I$ appears. Multiple applications of the identity (5.2) to the operator $\Delta_{j_{1}, k_{1}}(s / r) \ldots \Delta_{j_{l}, k_{l}}(s / r) T(\cdot)$ allow its expansion into a sum of operators each of which is a product of not less than $l \leq r$ of operators $T_{i}\left(\sigma_{i}\right)-I, \sigma_{i} \in(0, s / r), 1 \leq$ $i \leq n$. Consequently, (5.4) is dominated by a multiple of $s^{-l} \Omega^{l}(s, f)$. By summing the estimates obtained above we arrive at the inequality

$$
K\left(s^{r}, f, \mathbf{E}, \mathbf{E}^{r}\right) \leq C\left(\sum_{l=1}^{n} s^{r-l} \Omega^{l}(s, f)+s^{r}\|f\|_{\mathbf{E}}\right), \quad 0 \leq s \leq 1 .
$$

Note, that by repeating the known proof for the classical modulus of continuity one can prove the inequality

$$
\Omega^{l}(s, f) \leq C\left(s^{l}\|f\|_{\mathbf{E}}+s^{l} \int_{s}^{1} \sigma^{-1-l} \Omega^{k+r}(\sigma, f) d \sigma\right),
$$

which implies $s^{r-l} \Omega^{l}(s, f) \leq C\left(s^{r}\|f\|_{\mathbf{E}}+\Omega^{r}(s, f)\right)$. By applying this inequality to (5.5) and taking into account the inequality $K\left(s^{r}, f, \mathbf{E}, \mathbf{E}^{r}\right) \leq\|f\|$, we obtain the right-hand side of the estimate (1.7). To prove the left-hand side of (1.7) we first notice that the following inequality holds

$$
\Omega^{r}(s, g) \leq C s^{k} \sum_{1 \leq j_{1}, \ldots j_{k} \leq n} \Omega^{r-k}\left(s, D_{j_{1}} \ldots D_{j_{k}} g\right), g \in \mathbf{E}^{k}, C=C(k, r), k \leq r,
$$

which is an easy consequence of the identity (5.2) and the identity $\left(T_{j}(t)-I\right) g=$ $\int_{0}^{t} T_{j}(\tau) D_{j} g d \tau, g \in \mathcal{D}\left(D_{j}\right)$. From here, for any $f \in \mathbf{E}, g \in \mathbf{E}^{r}$ we obtain $\Omega^{r}(s, f) \leq$ $\Omega^{r}(s, f-g)+\Omega^{r}(s, g) \leq C\left(\|f-g\|_{\mathbf{E}}+s^{r}\|g\|_{\mathbf{E}^{r}}\right)$. The first item of Theorem 1.3 is proven and it obviously implies second item of the same Theorem.

REMARK 5.2. The proof shows that the left-hand side of (1.7) holds true for any finite set of one-parameter strongly continuous bounded semigroups.

Below is the proof of items (3)-(5) of Theorem 1.3 .

Proof. We will need the following lemma.

\section{LEMMA 5.3. The following inequalities hold}

$$
\begin{gathered}
\|f\|_{\mathbf{E}^{k}} \leq C\|f\|_{\mathbf{E}}^{1-k / r}\|f\|_{\mathbf{E}^{r}}^{k / r}, \quad f \in \mathbf{E}^{r}, \quad C=C(k, r), \\
K\left(s^{r}, f, \mathbf{E}, \mathbf{E}^{r}\right) \leq C s^{k}\|f\|_{\mathbf{E}^{k}}, \quad f \in \mathbf{E}^{k}, \quad C=C(k, r) .
\end{gathered}
$$

Proof. The first inequality follows from its well-known one-dimensional version (see also [33]). The second one follows from the right-hand estimate of (1.7) and (5.6).

This lemma shows that one can use the Reiteration Theorem (see [3, 10]), which immediately implies item (3) of Theorem [1.3. Next, let $\alpha>0,[\alpha]$ be a non-integer and its integer part respectively. According to item (3) of Theorem 1.3 we have $\left(\mathbf{E}, \mathbf{E}^{r}\right)_{\alpha / r, q}^{K}=\left(\mathbf{E}^{[\alpha]}, \mathbf{E}^{r}\right)_{(\alpha-[\alpha]) /(r-[\alpha]), q}^{K}$ and $\left(\mathbf{E}, \mathbf{E}^{1}\right)_{\alpha-[\alpha], q}^{K}=$ $\left(\mathbf{E}, \mathbf{E}^{r-[\alpha]}\right)_{(\alpha-[\alpha]) /(r-[\alpha]), q^{*}}^{K}$ Note, that $D_{j_{1}} D_{j_{2}} \ldots D_{j_{[\alpha]}}$ is a continuous map from 
$\left(\mathbf{E}^{[\alpha]}, \mathbf{E}^{r}\right)_{(\alpha-[\alpha]) /(r-[\alpha]), q}^{K}$ to $\left(\mathbf{E}, \mathbf{E}^{r-[\alpha]}\right)_{(\alpha-[\alpha]) /(r-[\alpha]), q}^{K}$. All together it shows that if $f \in\left(\mathbf{E}, \mathbf{E}^{r}\right)_{\alpha / r, q}^{K}$ then $D_{j_{1}} D_{j_{2}} \ldots D_{j_{[\alpha]}} f \in\left(\mathbf{E}, \mathbf{E}^{1}\right)_{\alpha-[\alpha], q}^{K}$ and

$$
\left\|D_{j_{1}} D_{j_{2}} \ldots D_{j_{[\alpha]}} f\right\|_{\left(\mathbf{E}, \mathbf{E}^{1}\right)_{\alpha-[\alpha], q}^{K}} \leq C\|f\|_{\left(\mathbf{E}, \mathbf{E}^{r}\right)_{\alpha / r, q}^{K}} .
$$

Conversely, let $D_{j_{1}} D_{j_{2}} \ldots D_{j_{[\alpha]}} f \in\left(\mathbf{E}, \mathbf{E}^{1}\right)_{\alpha-[\alpha], q}^{K}=\left(\mathbf{E}^{[\alpha]}, \mathbf{E}^{r}\right)_{(\alpha-[\alpha]) /(r-[\alpha]), q}^{K}$. Then the right-hand estimate of (1.7) and (5.6) imply

$$
\|f\|_{\left(\mathbf{E}, \mathbf{E}^{r}\right)_{\alpha / r, q}^{K}} \leq C \sum_{j_{1}, \ldots, j_{[\alpha]}=1}^{n}\left\|D_{j_{1}} D_{j_{2}} \ldots D_{j_{[\alpha]}} f\right\|_{\left(\mathbf{E}, \mathbf{E}^{1}\right)_{\alpha-[\alpha], q}^{K}} .
$$

Inequalities (5.9) and (5.10) imply item (4) of Theorem 1.3. Proof of item (5) is similar. Theorem 1.3 is completely proved.

\section{Shannon sampling, Paley-Wiener frames and abstract Besov subspaces}

6.1. Paley-Wiener vectors in Hilbert spaces. Consider a self-adjoint positive definite operator $L$ in a Hilbert space $\mathbf{H}$. Let $\sqrt{L}$ be the positive square root of $L$. According to the spectral theory for such operators $\mathbf{2}$ there exists a direct integral of Hilbert spaces $X=\int X(\lambda) d m(\lambda)$ and a unitary operator $\mathcal{F}$ from $\mathbf{H}$ onto $X$, which transforms the domains of $L^{k / 2}, k \in \mathbb{N}$, onto the sets $X_{k}=\left\{x \in X \mid \lambda^{k} x \in X\right\}$ with the norm

$$
\|x(\lambda)\|_{X_{k}}=\langle x(\lambda), x(\lambda)\rangle_{X(\lambda)}^{1 / 2}=\left(\int_{0}^{\infty} \lambda^{2 k}\|x(\lambda)\|_{X(\lambda)}^{2} d m(\lambda)\right)^{1 / 2} .
$$

and satisfies the identity $\mathcal{F}\left(L^{k / 2} f\right)(\lambda)=\lambda^{k}(\mathcal{F} f)(\lambda)$, if $f$ belongs to the domain of $L^{k / 2}$. We call the operator $\mathcal{F}$ the Spectral Fourier Transform [25, 26]. As known, $X$ is the set of all $m$-measurable functions $\lambda \mapsto x(\lambda) \in X(\lambda)$, for which the following norm is finite:

$$
\|x\|_{X}=\left(\int_{0}^{\infty}\|x(\lambda)\|_{X(\lambda)}^{2} d m(\lambda)\right)^{1 / 2}
$$

For a function $F$ on $[0, \infty)$ which is bounded and measurable with respect to $d m$ one can introduce the operator $F(\sqrt{L})$ by using the formula

$$
F(\sqrt{L}) f=\mathcal{F}^{-1} F(\lambda) \mathcal{F} f, \quad f \in \mathbf{H} .
$$

If $F$ is real-valued the operator $F(\sqrt{L})$ is self-adjoint.

REMARK 6.1. In many applications $L$ is a second-order differential operator and then $\sqrt{L}$ is a first-order pseudo-differential operator.

Definition 6.2. For $\sqrt{L}$ as above we will say that a vector $f \in \mathbf{H}$ belongs to the Paley-Wiener space $\mathbf{P} \mathbf{W}_{\omega}(\sqrt{L})$ if the support of the Spectral Fourier Transform $\mathcal{F} f$ is contained in $[0, \omega]$.

The next two facts are obvious.

TheORem 6.3. The spaces $\mathbf{P} \mathbf{W}_{\omega}(\sqrt{L})$ have the following properties:

(1) the space $\mathbf{P} \mathbf{W}_{\omega}(\sqrt{L})$ is a linear closed subspace in $\mathbf{H}$. 
(2) the space $\bigcup_{\omega>0} \mathbf{P W}_{\omega}(\sqrt{L})$ is dense in $\mathbf{H}$;

Next we denote by $\mathbf{H}^{k}$ the domain of $L^{k / 2}$. It is a Banach space, equipped with the graph norm $\|f\|_{k}=\|f\|_{\mathbf{H}}+\left\|L^{k / 2} f\right\|_{\mathbf{H}}$. The next theorem contains generalizations of several results from classical harmonic analysis (in particular the Paley-Wiener theorem). It follows from our results in [26.

TheOREM 6.4. The following statements hold:

(1) (Bernstein inequality) $f \in \mathbf{P W}_{\omega}(\sqrt{L})$ if and only if $f \in \mathbf{H}^{\infty}=\bigcap_{k=1}^{\infty} \mathbf{H}^{k}$, and the following Bernstein inequalities holds true

$$
\left\|L^{s / 2} f\right\|_{\mathbf{H}} \leq \omega^{s}\|f\|_{\mathbf{H}} \quad \text { for all } s \in \mathbb{R}_{+} ;
$$

(2) (Paley-Wiener theorem) $f \in \mathbf{P W}_{\omega}(\sqrt{L})$ if and only if for every $g \in \mathbf{H}$ the scalar-valued function of the real variable $t \mapsto\left\langle e^{i t \sqrt{L}} f, g\right\rangle$ is bounded on the real line and has an extension to the complex plane as an entire function of the exponential type $\omega$;

(3) (Riesz-Boas interpolation formula) $f \in \mathbf{P W}_{\omega}(\sqrt{L})$ if and only if $f \in \mathbf{H}^{\infty}$ and the following Riesz-Boas interpolation formula holds for all $\omega>0$ :

$$
i \sqrt{L} f=\frac{\omega}{\pi^{2}} \sum_{k \in \mathbb{Z}} \frac{(-1)^{k-1}}{(k-1 / 2)^{2}} e^{i\left(\frac{\pi}{\omega}(k-1 / 2)\right) \sqrt{L}} f .
$$

Proof. (1) follows immediately from the definition and representation (6.1). To prove (2) it is sufficient to apply the classical Bernstein inequality [16] in the uniform norm on $\mathbb{R}$ to every function $\left\langle e^{i t \sqrt{L}} f, g\right\rangle, g \in \mathbf{H}$. To prove (3) one has to apply the classical Riesz-Boas interpolation formula on $\mathbb{R},[\mathbf{3 6}, \mathbf{1 6}$, to a function $\left\langle e^{i t \sqrt{L}} f, g\right\rangle$.

6.2. Frames in Hilbert spaces. A family of vectors $\left\{\theta_{v}\right\}$ in a Hilbert space $\mathbf{H}$ is called a frame if there exist constants $A, B>0$ such that

$$
A\|f\|_{\mathbf{H}}^{2} \leq \sum_{v}\left|\left\langle f, \theta_{v}\right\rangle\right|^{2} \leq B\|f\|_{\mathbf{H}}^{2} \quad \text { for all } \quad f \in \mathbf{H} .
$$

The largest $A$ and smallest $B$ are called lower and upper frame bounds.

The family of scalars $\left\{\left\langle f, \theta_{v}\right\rangle\right\}$ represents a set of measurements of a vector $f$. In order to resynthesize the vector $f$ from this collection of measurements in a linear way one has to find another (dual) frame $\left\{\Theta_{v}\right\}$. Then a reconstruction formula is $f=\sum_{v}\left\langle f, \theta_{v}\right\rangle \Theta_{v}$. Dual frames are not unique in general. Moreover it may be difficult to find a dual frame in concrete situations. If $A=B=1$ the frame is said to be tight or Parseval. Parseval frames are similar in many respects to orthonormal wavelet bases. For example, if in addition all vectors $\theta_{v}$ are unit vectors, then the frame is an orthonormal basis. The main feature of Parseval frames is that decomposing and synthesizing a vector from known data are tasks carried out with the same family of functions, i.e., the Parseval frame is its own dual frame.

6.3. Sampling in abstract Paley-Wiener spaces. We now assume that the Assumption 3 is satisfied. It meant that there exists a $C>0$ and $m_{0} \geq 0$ such that for any $0<\rho<1$ there exists a set of functionals $\mathcal{A}^{(\rho)}=\left\{\mathcal{A}_{k}^{(\rho)}\right\}$, defined on $\mathbf{H}^{m_{0}}$, for which the inequalties (1.11) hold true. 
Remark 6.5. Following [27, 29] we call inequality (1.11) a Poincaré-type inequality since it is an estimate of the norm of $f$ through the norm of its "derivative" $L^{m / 2} f$.

Let us introduce vectors $\mu_{k} \in \mathbf{H}$ such that $\left\langle f, \mu_{k}\right\rangle=\mathcal{A}_{k}^{(\rho)}(f), f \in \mathbf{H}^{m}, m>m_{0}$. Let $\mathcal{P}_{\omega}$ be the orthogonal projection of $\mathbf{H}$ onto $\mathbf{P W}_{\omega}(\sqrt{L})$ and put

$$
\phi_{k}^{\omega}=\mathcal{P}_{\omega} \mu_{k} .
$$

Using the Bernstein inequality (6.3) we obtain the following statement.

Theorem 6.6. (Sampling Theorem) Assume that inequality (1.11) holds and for a given $\omega>0$ and $\delta \in(0,1)$ pick a $\rho$ such that

$$
\rho^{2 m}=C^{-1} \omega^{-2 m} \delta .
$$

Then the family of vectors $\left\{\phi_{k}^{\omega}\right\}$ is a frame for the Hilbert space $\mathbf{P} \mathbf{W}_{\omega}(\sqrt{L})$ and

$$
(1-\delta)\|f\|_{\mathbf{H}}^{2} \leq \sum_{k}\left|\left\langle f, \phi_{k}^{\omega}\right\rangle\right|^{2} \leq\|f\|_{\mathbf{H}}^{2}, \quad f \in \mathbf{P W}_{\omega}(\sqrt{L}) .
$$

The canonical dual frame $\left\{\Theta_{k}^{\omega}\right\}$ has the property $\Theta_{k}^{\omega} \in \mathbf{P W}_{\omega}(\sqrt{L})$ and provides the following reconstruction formulas

$$
f=\sum_{k}\left\langle f, \phi_{k}^{\omega}\right\rangle \Theta_{k}^{\omega}=\sum_{k}\left\langle f, \Theta_{k}^{\omega}\right\rangle \phi_{k}^{\omega}, \quad f \in \mathbf{P W}_{\omega}(\sqrt{L}) .
$$

6.4. Partitions of unity on the frequency side. The construction of frequencylocalized frames is achieved via spectral calculus. The idea is to start from a partition of unity on the positive real axis. In the following, we will be considering two different types of such partitions, whose construction we now describe in some detail.

Let $g \in C^{\infty}\left(\mathbb{R}_{+}\right)$be a non-increasing function such that $\operatorname{supp}(g) \subset[0,2]$, and $g(\lambda)=1$ for $\lambda \in[0,1], 0 \leq g(\lambda) \leq 1, \lambda>0$. We now let $h(\lambda)=g(\lambda)-g(2 \lambda)$, which entails $\operatorname{supp}(h) \subset\left[2^{-1}, 2\right]$, and use this to define $F_{0}(\lambda)=\sqrt{g(\lambda)}, F_{j}(\lambda)=$ $\sqrt{h\left(2^{-j} \lambda\right)}, j \geq 1$, as well as $G_{j}(\lambda)=\left[F_{j}(\lambda)\right]^{2}=F_{j}^{2}(\lambda), j \geq 0$. As a result of the definitions, we get for all $\lambda \geq 0$ the equations $\sum_{j=0}^{n} G_{j}(\lambda)=\sum_{j=0}^{n} F_{j}^{2}(\lambda)=$ $g\left(2^{-n} \lambda\right)$, and as a consequence $\sum_{j \geq 0} G_{j}(\lambda)=\sum_{j \geq 0} F_{j}^{2}(\lambda)=1, \quad \lambda \geq 0$, with finitely many nonzero terms occurring in the sums for each fixed $\lambda$. We call the sequence $\left(G_{j}\right)_{j \geq 0}$ a (dyadic) partition of unity, and $\left(F_{j}\right)_{j \geq 0}$ a quadratic (dyadic) partition of unity. As will become soon apparent, quadratic partitions are useful for the construction of frames. Using the spectral theorem one has $F_{j}^{2}(\sqrt{L}) f=\mathcal{F}^{-1}\left(F_{j}^{2}(\lambda) \mathcal{F} f(\lambda)\right), \quad j \geq 1$, and thus

$$
f=\mathcal{F}^{-1} \mathcal{F} f(\lambda)=\mathcal{F}^{-1}\left(\sum_{j \geq 0} F_{j}^{2}(\lambda) \mathcal{F} f(\lambda)\right)=\sum_{j \geq 0} F_{j}^{2}(\sqrt{L}) f
$$

Taking inner product with $f$ gives $\left\|F_{j}(\sqrt{L}) f\right\|_{\mathbf{H}}^{2}=\left\langle F_{j}^{2}(\sqrt{L}) f, f\right\rangle$ and $\|f\|_{\mathbf{H}}^{2}=$ $\sum_{j \geq 0}\left\langle F_{j}^{2}(\sqrt{L}) f, f\right\rangle=\sum_{j \geq 0}\left\|F_{j}(\sqrt{L}) f\right\|_{\mathbf{H}}^{2}$. Similarly, we get the identity $\sum_{j \geq 0} G_{j}(\sqrt{L}) f=$ $f$. Moreover, since the functions $G_{j}, F_{j}$, have their supports in $\left[2^{j-1}, 2^{j+1}\right]$, the elements $F_{j}(\sqrt{L}) f$ and $G_{j}(\sqrt{L}) f$ are bandlimited to $\left[2^{j-1}, 2^{j+1}\right]$, whenever $j \geq 1$, and to $[0,2]$ for $j=0$. 
6.5. Paley-Wiener frames in Hilbert spaces. Using the notation from above and Theorem 6.6, one can describe the following Paley-Wiener frame in an abstract Hilbert space $\mathbf{H}$.

Theorem 6.7. (Paley-Wiener nearly Parseval frame in $\mathbf{H}$ )

For a fixed $\delta \in(0,1)$ and $j \in \mathbb{N}$ let $\left\{\phi_{k}^{j}\right\}$ be a set of vectors described in Theorem 6.6 that correspond to $\omega=2^{j+1}$. Then for functions $F_{j}$ the family of Paley-Wiener vectors $\Phi_{k}^{j}=F_{j}(\sqrt{L}) \phi_{k}^{j}$ has the following properties:

(1) Each vector $\Phi_{k}^{j}$ belongs to $\mathbf{P W}_{\left[2^{j-1}, 2^{j+1}\right]}(\sqrt{L}), j \in N, k=1, \ldots$

(2) The family $\left\{\Phi_{k}^{j}\right\}$ is a frame in $\mathbf{H}$ with constants $1-\delta$ and 1 :

$$
(1-\delta)\|f\|_{\mathbf{H}}^{2} \leq \sum_{j \geq 0} \sum_{k}\left|\left\langle f, \Phi_{k}^{j}\right\rangle\right|^{2} \leq\|f\|_{\mathbf{H}}^{2}, \quad f \in \mathbf{H} .
$$

(3) The canonical dual frame $\left\{\Psi_{k}^{j}\right\}$ also consists of bandlimited vectors $\Psi_{k}^{j} \in$ $\mathbf{P W}_{\left[2^{j-1}, 2^{j+1}\right]}(\sqrt{L}), j \in[0, \infty), k=1, \ldots$, and has frame bounds $A=$ $1, B=(1-\delta)^{-1}$.

(4) The reconstruction formulas hold for every $f \in \mathbf{H}$

$$
f=\sum_{j} \sum_{k}\left\langle f, \Phi_{k}^{j}\right\rangle \Psi_{k}^{j}=\sum_{j} \sum_{k}\left\langle f, \Psi_{k}^{j}\right\rangle \Phi_{k}^{j} .
$$

The last two items here follow from the first two and general properties of frames. We also note that for reconstruction of a Paley-Wiener vector from a set of samples one can use, besides dual frames, the variational (polyharmonic) splines in Hilbert spaces developed in [27.

\section{Besov subspaces in Hilbert spaces}

7.1. Approximation spaces. Let us introduce another functional on $\mathbf{E}+\mathbf{F}$, where $\mathbf{E}$ and $\mathbf{F}$ form a pair of quasi-normed linear spaces $\mathcal{E}(f, t)=\mathcal{E}(f, t, \mathbf{E}, \mathbf{F})=$ $\inf _{g \in \mathbf{F},\|g\|_{\mathbf{F}} \leq t}\|f-g\|_{\mathbf{E}}$.

Definition 7.1. The approximation space $\mathcal{E}_{\alpha, q}(\mathbf{E}, \mathbf{F}), 0<\alpha<\infty, 0<q \leq \infty$ is the quasi-normed linear spaces of all $f \in \mathbf{E}+\mathbf{F}$ for which the quasi-norm

$$
\|f\|_{\mathcal{E}_{\alpha, q}(\mathbf{E}, \mathbf{F})}=\left(\int_{0}^{\infty}\left(t^{\alpha} \mathcal{E}(f, t)\right)^{q} \frac{d t}{t}\right)^{1 / q}
$$

is finite.

The next theorem represents a very abstract version of what is known as an Equivalence Approximation Theorem [18, 2]. In the form it is stated below it was proved in $[\mathbf{9}$.

THEOREM 7.2. Suppose that $\mathcal{T} \subset \mathbf{F} \subset \mathbf{E}$ are quasi-normed linear spaces and $\mathbf{E}$ and $\mathbf{F}$ are complete. If there exist $C>0$ and $\beta>0$ such that the following Jackson-type inequality is satisfied $t^{\beta} \mathcal{E}(t, f, \mathcal{T}, \mathbf{E}) \leq C\|f\|_{\mathbf{F}}, t>0, f \in \mathbf{F}$, then the following embedding holds true

$$
(\mathbf{E}, \mathbf{F})_{\theta, q}^{K} \subset \mathcal{E}_{\theta \beta, q}(\mathbf{E}, \mathcal{T}), \quad 0<\theta<1,0<q \leq \infty .
$$

If there exist $C>0$ and $\beta>0$ such that the following Bernstein-type inequality holds $\|f\|_{\mathbf{F}} \leq C\|f\|_{\mathcal{T}}^{\beta}\|f\|_{\mathbf{E}}, f \in \mathcal{T}$, then the following embedding holds true

$$
\mathcal{E}_{\theta \beta, q}(\mathbf{E}, \mathcal{T}) \subset(\mathbf{E}, \mathbf{F})_{\theta, q}^{K}, \quad 0<\theta<1,0<q \leq \infty .
$$


7.2. Besov subspaces in Hilbert spaces. According to (1.6) we introduce $\mathcal{B}_{\mathbf{H}, q}^{\alpha}(\sqrt{L})=\left(\mathbf{H}, \mathbf{H}^{r}\right)_{\theta, q}^{K}, \quad 0<\theta=\alpha / r<1, \quad 1 \leq q \leq \infty$. We also introduce a notion of best approximation:

$$
\mathcal{E}(f, \omega)=\inf _{g \in \mathbf{P W}_{\omega}(\sqrt{L})}\|f-g\|_{\mathbf{H}} .
$$

Our goal is to apply Theorem 7.2 in the situation where $\mathbf{E}=\mathbf{H}, \quad \mathbf{F}=\mathbf{H}^{r}$ and $\mathcal{T}=\mathbf{P} \mathbf{W}_{\omega}(\sqrt{L})$ is a natural abelian group as the additive group of a vector space, with the quasi-norm $\|f\|_{\mathcal{T}}=\inf \left\{\omega^{\prime}>0: f \in \mathbf{P W}_{\omega^{\prime}}(\sqrt{L})\right\}$. To be more precise it is the space of finite sequences of Fourier coefficients $\mathbf{c}=$ $\left(c_{1}, \ldots c_{m}\right) \in \mathbf{P W}_{\omega}(\sqrt{L})$ where $m$ is the greatest index such that the eigenvalue $\lambda_{m} \leq \omega$. For a $\mathbf{c}=\left(c_{1}, \ldots c_{m}\right) \in \mathbf{P} \mathbf{W}_{\omega}(\sqrt{L})$ the quasi-norm is defined as $\|\mathbf{c}\|_{E_{\omega}(L)}=$ $\max \left\{\sqrt{\lambda_{j}}: c_{j} \neq 0, c_{j+1}=\ldots=c_{m}=0\right\}$.

REMARK 7.3. Let us emphasize that the reason we need the language of quasi-normed spaces is because $\|\cdot\|_{\mathcal{T}}$ is clearly not a norm, only a quasi-norm on $\mathbf{P W}_{\omega}(\sqrt{L})$.

The Plancherel Theorem allows us to verify a generalization of the Bernstein inequality for bandlimited functions in $f \in \mathbf{P W}_{\omega}(\sqrt{L})$. One can prove the following statement (see [22, 26]).

Lemma 7.4. A vector $f$ belongs to the space $\mathbf{P} \mathbf{W}_{\omega}(\sqrt{L})$ if and only if the following Bernstein inequality holds $\left\|L^{r / 2} f\right\|_{\mathbf{H}} \leq \omega^{r}\|f\|_{\mathbf{H}}, \quad r \in \mathbb{R}_{+}$.

One also has an analogue of the Jackson inequality (see [22, [26] $) \mathcal{E}(f, \omega) \leq$ $\omega^{-r}\|f\|_{\mathbf{H}^{r}}, \quad f \in \mathbf{H}^{r}$. These two inequalities and Theorem 7.2 imply the following result (compare to [31, [34]).

Theorem 7.5. For $\alpha>0,1 \leq q \leq \infty$ the norm of $\mathcal{B}_{\mathbf{H}, q}^{\alpha}(\sqrt{L})$, is equivalent to

$$
\|f\|_{\mathbf{H}}+\left(\sum_{j=0}^{\infty}\left(2^{j \alpha} \mathcal{E}\left(f, 2^{j}\right)\right)^{q}\right)^{1 / q} .
$$

Let the functions $F_{j}$ be as in Subsection 6.4

Theorem 7.6. For $\alpha>0,1 \leq q \leq \infty$ the norm of $\mathcal{B}_{\mathbf{H}, q}^{\alpha}(\sqrt{L})$, is equivalent to

$$
f \mapsto\left(\sum_{j=0}^{\infty}\left(2^{j \alpha}\left\|F_{j}(\sqrt{L}) f\right\|_{\mathbf{H}}\right)^{q}\right)^{1 / q},
$$

with the standard modifications for $q=\infty$.

Proof. We obviously have $\mathcal{E}\left(f, 2^{l}\right) \leq \sum_{j>l}\left\|F_{j}(\sqrt{L}) f\right\|_{\mathbf{H}}$. By using a discrete version of Hardy's inequality [3] we obtain the estimate

$$
\|f\|+\left(\sum_{l=0}^{\infty}\left(2^{l \alpha} \mathcal{E}\left(f, 2^{l}\right)\right)^{q}\right)^{1 / q} \leq C\left(\sum_{j=0}^{\infty}\left(2^{j \alpha}\left\|F_{j}(\sqrt{L}) f\right\|_{\mathbf{H}}\right)^{q}\right)^{1 / q} .
$$


Conversely, for any $g \in \mathbf{P} \mathbf{W}_{2^{j-1}}(\sqrt{L})$ we have $\left\|F_{j}(\sqrt{L}) f\right\|_{\mathbf{H}}=\left\|F_{j}(\sqrt{L})(f-g)\right\|_{\mathbf{H}} \leq$ $\|f-g\|_{\mathbf{H}}$. This implies the estimate $\left\|F_{j}(\sqrt{L}) f\right\|_{\mathbf{H}} \leq \mathcal{E}\left(f, 2^{j-1}\right)$, which shows that the inequality opposite to (7.6) holds. The proof is complete.

TheOREM 7.7. For $\alpha>0,1 \leq q \leq \infty$ the norm of $\mathcal{B}_{\mathbf{H}, q}^{\alpha}(\sqrt{L})$ is equivalent to

$$
\left(\sum_{j=0}^{\infty} 2^{j \alpha q}\left(\sum_{k}\left|\left\langle f, \Phi_{k}^{j}\right\rangle\right|^{2}\right)^{q / 2}\right)^{1 / q} \asymp\|f\|_{B_{q}^{\alpha}},
$$

with the standard modifications for $q=\infty$.

Proof. For $f \in \mathbf{H}$ and operator $F_{j}(\sqrt{L})$ we apply (6.8) to $F_{j}(\sqrt{L}) f \in \mathbf{P W}_{2^{j+1}}(\sqrt{L})$ to obtain

$$
(1-\delta)\left\|F_{j}(\sqrt{L}) f\right\|_{\mathbf{H}}^{2} \leq \sum_{k}\left|\left\langle F_{j}(\sqrt{L}) f, \phi_{k}^{j}\right\rangle\right|^{2} \leq\left\|F_{j}(\sqrt{L}) f\right\|_{\mathbf{H}}^{2} .
$$

Since $\Phi_{k}^{j}=F_{j}(\sqrt{L}) \phi_{k}^{j}$ we obtain the following inequality

$$
\sum_{k}\left|\left\langle f, \Phi_{k}^{j}\right\rangle\right|^{2} \leq\left\|F_{j}(\sqrt{L}) f\right\|_{\mathbf{H}}^{2} \leq \frac{1}{1-\delta} \sum_{k}\left|\left\langle f, \Phi_{k}^{j}\right\rangle\right|^{2} \quad \text { for all } f \in \mathbf{H} .
$$

Our statement follows now from Theorem 7.6 ,

$$
\begin{aligned}
& =\mathrm{I}=\mathrm{=}=\mathrm{=}=\mathrm{=}=\mathrm{=}=\mathrm{=}=\mathrm{=}=\mathrm{=}=\mathrm{=}=\mathrm{=} \\
& =\mathrm{I}=\mathrm{=}=\mathrm{=}=\mathrm{=}=\mathrm{=}=\mathrm{=}=\mathrm{=}=\mathrm{=}=\mathrm{=}=\mathrm{=}=\mathrm{=}
\end{aligned}
$$

\section{Applications}

8.1. Analysis on $\mathbb{S}^{d}$. We will specify the general setup in the case of standard unit sphere. Let $\mathbb{S}^{d}=\left\{x \in \mathbb{R}^{d+1}:\|x\|=1\right\}$. Let $\mathcal{P}_{n}$ denote the space of spherical harmonics of degree $n$, which are restrictions to $\mathbb{S}^{d}$ of harmonic homogeneous polynomials of degree $n$ in $\mathbb{R}^{d}$. The Laplace-Beltrami operator $\Delta_{\mathbb{S}^{d}}$ on $\mathbb{S}^{d}$ is a restriction of the regular Laplace operator $\Delta$ in $\mathbb{R}^{d}$. Namely, $\Delta_{\mathbb{S}^{d}} f(x)=\Delta \widetilde{f}(x), x \in \mathbb{S}^{d}$, where $\widetilde{f}(x)$ is the homogeneous extension of $f: \tilde{f}(x)=f(x /\|x\|)$. Another way to compute $\Delta_{\mathbb{S}^{d}} f(x)$ is to express both $\Delta_{\mathbb{S}^{d}}$ and $f$ in a spherical coordinate system. Each $\mathcal{P}_{n}$ is the eigenspace of $\Delta_{\mathbb{S}^{d}}$ that corresponds to the eigenvalue $-n(n+d-1)$. Let $Y_{n, l}, l=1, \ldots, l_{n}$ be an orthonormal basis in $\mathcal{P}_{n}$.

Let $e_{1}, \ldots, e_{d+1}$ be the standard orthonormal basis in $\mathbb{R}^{d+1}$. If $S O(d+1)$ and $S O(d)$ are the groups of rotations of $\mathbb{R}^{d+1}$ and $\mathbb{R}^{d}$ respectively then $\mathbb{S}^{d}=S O(d+$ 1) $/ S O(d)$. On $\mathbb{S}^{d}$ we consider vector fields $X_{i, j}=x_{j} \partial_{x_{i}}-x_{i} \partial_{x_{j}}$ which are generators of one-parameter groups of rotations $\exp t X_{i, j} \in S O(d+1)$ in the plane $\left(x_{i}, x_{j}\right)$. These groups are defined by the formulas for $\tau \in \mathbb{R}$,

$\exp \tau X_{i, j} \cdot\left(x_{1}, \ldots, x_{d+1}\right)=\left(x_{1}, \ldots, x_{i} \cos \tau-x_{j} \sin \tau, \ldots, x_{i} \sin \tau+x_{j} \cos \tau, \ldots, x_{d+1}\right)$

Let $T_{i, j}(\tau)$ be a one-parameter group which is a representation of $\exp \tau X_{i, j}$ in the space $L_{p}\left(\mathbb{S}^{d}\right)$. It acts on $f \in L_{p}\left(\mathbb{S}^{d}\right)$ by the following formula

$$
T_{i, j}(\tau) f\left(x_{1}, \ldots, x_{d+1}\right)=f\left(x_{1}, \ldots, x_{i} \cos \tau-x_{j} \sin \tau, \ldots, x_{i} \sin \tau+x_{j} \cos \tau, \ldots, x_{d+1}\right) .
$$

Let $D_{i, j}$ be a generator of $T_{i, j}$ in $L_{p}\left(\mathbb{S}^{d}\right)$. The Laplace-Beltrami operator $\Delta_{\mathbb{S}^{d}}$ can be identified with the operator $L=\sum_{i<j} D_{i, j}^{2}$. One can easily illustrate our results by describing norms in Sobolev and Besov spaces on $\mathbb{S}^{d}$ in terms of operators $T_{i, j}$ 
and $D_{i, j}$. In this situation role of Paley-Wiener subspaces is played by subspaces $\mathcal{P}_{n}$. A set of functionals $\left\{\mathcal{A}_{k}^{(\rho)}\right\}_{k \in \mathcal{K}_{\rho}}$ described in (1.11), (6.7) can be represented by a set of Dirac measures at nodes $\left\{x_{k}^{(\rho)}\right\}$ "nearly uniformly" distributed over the sphere $\mathbb{S}^{d}$.

8.2. Compact homogeneous manifolds. It should be noted, that a similar situation holds on any compact homogeneous manifolds $M=G / K$ where $G$ is a compact Lie group and $K$ is its closed subgroup. Moreover, in this case description of Besov spaces in terms of approximation by Paley-Wiener vectors (eigenfunctions of a corresponding Laplace-Beltrami operator) and in terms of frame coefficients can be extended to any $1 \leq p \leq \infty\left[\mathbf{7},\left[38\right.\right.$, , 6]. A set of functionals $\left\{\mathcal{A}_{k}^{(\rho)}\right\}_{k \in \mathcal{K}_{\rho}}$ can be represented by a set of Dirac measures (or some other functionals [29]) at a set of nodes $\left\{x_{k}^{(\rho)}\right\}$ "nearly uniformly" distributed over the manifold $M$ with the spacing comparable to $\rho>0$. The Weyl's asymptotic formula 8 implies [28] that a rate of sampling which is given by (6.7) is essentially optimal.

8.3. Non-compact symmetric spaces. Our framework also holds on noncompact symmetric spaces [30]-35. Besov spaces can be characterized either using corresponding modulus of continuity $\mathbf{3 2}$ or by approximation by Paley-Wiener vectors [33] or in terms of frames [35. In this situation Paley-Wiener functions which admit explicit description in terms of the Helgason-Fourier transform 33 can be represented by a set of Dirac measures (or even more general functionals [29]) at a set of nodes $\left\{x_{k}^{(\rho)}\right\}$ "nearly uniformly" distributed over the manifold $M$ with the spacing comparable to $\rho>0$.

REMARK 8.1. It should be noted that in the case of non-compact symmetric spaces our approach leads to Sobolev and Besov spaces which are different from the conventional ones generated by the Laplace-Beltrami operator associated with the natural metric.

\section{References}

[1] J. Bergh, J. Lofstrom, Interpolation spaces, Springer-Verlag, 1976.

[2] M. Birman and M. Solomyak, Spectral theory of selfadjoint operators in Hilbert space, D. Reidel Publishing Co., Dordrecht, 1987.

[3] P. Butzer, H. Berens, Semi-Groups of operators and approximation, Springer, Berlin, 1967.

[4] P. L. Butzer, K. Scherer, Jackson and Bernstein-type inequalities for families of commutative operators in Banach spaces, J. Approx. Theory 5 (1972), 308-342.

[5] Dai, Feng; Xu, Yuan, Approximation theory and harmonic analysis on spheres and balls, Springer Monographs in Mathematics. Springer, New York, 2013. xviii+440 pp. ISBN: 978-14614-6659-8; 978-1-4614-6660-4

[6] H. G. Feichtinger, H. Führ, I. Z. Pesenson, Geometric Space-Frequency Analysis on Manifolds, Journal of Fourier Analysis and Applications, December 2016, Volume 22, Issue 6, pp 12941355.

[7] D. Geller and I. Pesenson, Band-limited localized Parseval frames and Besov spaces on compact homogeneous manifolds, J. Geom. Anal. 21/2 (2011), 334-371.

[8] L. Hörmander, The Analysis of Linear Partial Differential Operators. III. Pseudo-differential Operators, Springer, Berlin, 2007.

[9] S. Krein, I. Pesenson, Interpolation Spaces and Approximation on Lie Groups, The Voronezh State University, Voronezh, 1990, 
[10] S. Krein, Y. Petunin, E. Semenov, Interpolation of linear operators, Translations of Mathematical Monographs, 54. AMS, Providence, R.I., 1982.

[11] J. Lions, Theorems de trace et d'interpolation, Ann. Scuola Norm.Sup.Pisa (3)13, 389-403 (1959).

[12] J. Lions, J. Peetre, Sur one classe d'espaces d'interpolation, Inst. Hautes Etudes Sci. Publ. Math. 19, 5-68 (1964).

[13] E. Nelson, Analytic vectors, Ann. of Math., 70(3), (1959), 572-615.

[14] E. Nelson, W. Stinespring, Representation of elliptic operators in an enveloping algebra, Amer. J. Math. 81 (1959), 547-560.

[15] Müller, Detlef; Yang, Dachun A difference characterization of Besov and Triebel-Lizorkin spaces on RD-spaces. Forum Math. 21 (2009), no. 2, 259-298.

[16] S. M. Nikolskii, Approximation of functions of several variables and imbedding theorems, Springer, Berlin, 1975.

[17] Nursultanov, Erlan; Ruzhansky, Michael; Tikhonov, Sergey, Nikolskii inequality and Besov, Triebel-Lizorkin, Wiener and Beurling spaces on compact homogeneous manifolds, Ann. Sc. Norm. Super. Pisa Cl. Sci. (5) 16 (2016), no. 3, 981-1017.

[18] J. Peetre, G. Sparr, Interpolation on normed Abelian groups, Ann. Mat. Pura Appl. 92 (1972), 217-262.

[19] Pesenson, I. Z., Interpolation of noncommuting operators, (Russian) Uspehi Mat. Nauk 33 (1978), no. 3(201), 183-184.

[20] I. Pesenson, Interpolation spaces on Lie groups, (Russian) Dokl. Akad. Nauk SSSR 246 (1979), no. 6, 1298-1303.

[21] I. Pesenson, Nikolskii-Besov spaces connected with representations of Lie groups, (Russian) Dokl. Akad. Nauk SSSR 273 (1983), no. 1, 45-49.

[22] I. Pesenson, The Best Approximation in a Representation Space of a Lie Group, Dokl. Acad. Nauk USSR, v. 302, No 5, pp. 1055-1059, (1988) (Engl. Transl. in Soviet Math. Dokl., v.38, No 2, pp. 384-388, 1989.)

[23] I. Pesenson, On the abstract theory of Nikolskii-Besov spaces, (Russian) Izv. Vyssh. Uchebn. Zaved. Mat. 1988, no. 6, 59-68; translation in Soviet Math. (Iz. VUZ) 32 (1988), no. 6, 80Đ92

[24] I. Pesenson, Approximations in the representation space of a Lie group, (Russian) Izv. Vyssh. Uchebn. Zaved. Mat. 1990, no. 7, 43-50; translation in Soviet Math. (Iz. VUZ) 34 (1990), no. 7, 49 Đ57.

[25] I. Pesenson, The Bernstein Inequality in the Space of Representation of Lie group, Dokl. Acad. Nauk USSR 313 (1990), 86-90; English transl. in Soviet Math. Dokl. 42 (1991).

[26] I. Pesenson, A sampling theorem on homogeneous manifolds, Trans. Amer. Math. Soc. 352/9 (2000), 4257-4269.

[27] I. Pesenson, Sampling of band limited vectors, J. Fourier Anal. Appl. $7 / 1$ (2001), 93-100.

[28] I. Pesenson, An approach to spectral problems on Riemannian manifolds, Pacific J. Math. 215/1 (2004), 183-199.

[29] I. Pesenson, Poincaré-type inequalities and reconstruction of Paley-Wiener functions on manifolds, J. Geometric Anal. 4/1 (2004), 101-121.

[30] I. Pesenson, Bernstein-Nikolski inequality and Riesz interpolation formula on compact homogeneous manifolds, J. Approx. Theory, 150/2 (2008), 175-198.

[31] I. Pesenson, Paley-Wiener approximations and multiscale approximations in Sobolev and Besov spaces on manifolds, J. Geom. Anal. 19 (2009), no. 2, 390Đ419.

[32] I. Z. Pesenson, Bernstein-Nikolskii and Plancherel-Polya inequalities in Lp-norms on noncompact symmetric spaces, Math. Nachr. 282/2 (2009), 253-269.

[33] I. Pesenson, A Discrete Helgason-Fourier transform for Sobolev and Besov functions on noncompact symmetric spaces, Contemp. Math. 464, Amer. Math. Soc. (2008), 231-249.

[34] I. Z. Pesenson, M. Z. Pesenson, Approximation of Besov vectors by Paley-Wiener vectors in Hilbert spaces, Approximation Theory XIII: San Antonio 2010 (Springer Proceedings in Mathematics), by Marian Neamtu and Larry Schumaker, 249-263.

[35] I. Z. Pesenson, Paley-Wiener-Schwartz nearly Parseval frames on noncompact symmetric spaces, Commutative and Noncommutative Harmonic Analysis and Applications, 55-71, Contemp. Math. 603, Amer. Math. Soc., Providence, RI, 2013.

[36] I. Z. Pesenson, Boas-type formulas and sampling in Banach spaces with applications to analysis on manifolds, in New Perspectives on Approximation and Sampling Theory, Springer International Publishing, Switzerland (2014), 39 -61. 
[37] I. Z. Pesenson, Splines and Wavelets on Geophysically Relevant Manifolds, Handbook of Geomathematics, Springer, Berlin (2014), 1-33.

[38] I. Z. Pesenson, Approximations in $L_{p}$-norms and Besov spaces on compact manifolds, Contemporary Mathematics, 650 (2015), 199-210.

[39] Pesenson, Isaac Z. Sampling, splines and frames on compact manifolds. GEM Int. J. Geomath. 6 (2015), no. $1,43-81$.

Department of Mathematics, Temple University, Philadelphia, PA 19122

Email address: pesenson@temple.edu 